\title{
COULOMB ENERGIES AND THE SHELL MODEL
}

\author{
K. ASHKTORAB ${ }^{1}$, S. ELITZUR ${ }^{2}$, J. JÄNECKE ${ }^{1}$, S. LIRAN $^{2, *}$ and N. ZELDES ${ }^{1,2, \star \star}$ \\ ${ }^{1}$ Department of Physics, The University of Michigan, Ann Arbor, MI 48109-1090, USA \\ ${ }^{2}$ The Racah Institute of Physics, The Hebrew University of Jerusalem, Jerusalem 91904, Israel
}

\author{
Received 12 December 1989 \\ (Revised 7 May 1990)
}

\begin{abstract}
Phenomenological shell-model Coulomb energy equations with coefficients varying as $A^{-1 / 3}$ are considered. The highly regular systematics of Coulomb displacement energies is presented and discussed. In a global fit with 22 adjustable parameters the equations reproduce 377 displacement energies with a standard deviation of $55 \mathrm{keV}$. The relations to other shell-model equations are discussed. The numerical values of the adjusted coefficients are related to the charge radius and deformation parameters of the liquid drop model. The calculation of total Coulomb energies for ground states is considered.
\end{abstract}

\section{Introduction}

The total energy of the nucleus is a sum of nuclear and Coulomb energies. In a semi-empirical shell-model treatment the parameters describing them are statistically correlated. In order to obtain physically meaningful and statistically significant coefficients for mass equations one has to consider both ground state masses and Coulomb displacement energies (CDE). This was one of the main motivations for the present study.

In the mass equation of ref. ${ }^{1}$ ) Coulomb energy coefficients were determined by using the Carlson-Talmi Coulomb energy equation ${ }^{2,3}$ ) both for the ground state and for excited isobaric analog states (IAS), with coefficients varying like $A^{-1 / 3}$. The adjusted coefficients described ${ }^{4}$ ) experimental Coulomb displacement energies with a standard deviation of about one third of the deviation obtained for the masses. On the other hand, in the above procedure one neglects the dependence of the Coulomb energy in a given nucleus on the isospin $T$.

In ref. ${ }^{5}$ ) the $T$-dependence of Coulomb displacement energies was calculated assuming the Carlson-Talmi equation for ground states. A variation of the coefficients with nucleon numbers was adopted, which reflects upon the isotope shifts of nuclear charge radii. The resulting equations were adjusted to the data simultaneously in all shell regions, resulting in a mean deviation smaller than that for the Carlson-Talmi equation.

* Present address: 17 Einstein St., Haifa 34605, Israel.

* Permanent address: The Racah Institute of Physics, The Hebrew University of Jerusalem, Jerusalem 91904, Israel. 
In the present work, which continues the studies started in ref. ${ }^{5}$ ), the complete equations of ref. $\left.{ }^{5}\right)^{\star}$ are applied to presently available experimental CDE. A simple $A^{-1 / 3}$ scaling of the coefficients is used to facilitate combining the results with the mass equation of ref. ${ }^{1}$ ). As in ref. ${ }^{5}$ ), charge-dependent nuclear effects and the electromagnetic spin-orbit interaction are not considered.

The work is organized as follows: in sect. 2 we summarize and make some comments on the equations, using the notation of ref. ${ }^{1}$ ). In sect. 3 the very regular isodiapheric (constant $N-Z$ ) systematics of Coulomb displacement energies is presented and discussed. Numerical results of the adjustment process are given and discussed in sect. 4. Their relations to geometrical parameters of the liquid drop model are discussed phenomenologically in sect. 5. Calculation of total ground-state Coulomb energies is considered in sect. 6 . The conclusions are summarized in sect. 7 . Calculational details and a table of recent experimental Coulomb displacement energies are given in an appendix.

\section{Coulomb energy equations}

The Coulomb energy of isobaric analog members of an isospin multiplet and their Coulomb displacement energies between neighboring members are, respectively, given by the isobaric multiplet mass equation (IMME) ${ }^{6.7}$ ) as

$$
\begin{gathered}
\mathrm{E}^{\mathrm{C}}\left(A \alpha T, T_{z}\right)=E^{\mathrm{C}(0)}(A \alpha T)-T_{z} E^{\mathrm{C}(1)}(A \alpha T)+\left(3 T_{z}^{2}-T(T+1)\right) E^{\mathrm{C}(2)}(A \alpha T), \\
\Delta E^{\mathrm{C}}\left(A \alpha T, T_{z}\right)=E^{\mathrm{C}(1)}(A \alpha T)-3\left(2 T_{z}-1\right) E^{\mathrm{C}(2)}(A \alpha T) .
\end{gathered}
$$

We use the usual notation $N, Z$ and $A=N+Z$ to denote neutron, proton and total nucleon numbers and $T$ and $T_{z}=\frac{1}{2}(N-Z)$ to denote the quantum numbers of the total isospin and its $z$-component. The $\alpha$ denote quantum numbers needed additionally to $A, T$ and $T_{z}$ for a complete characterization of the states. The coefficients $E^{C(0)}, E^{C(1)}$ and $E^{C(2)}$ are the isoscalar, isovector and isotensor Coulomb energies of the multiplet.

In this work, like in refs. ${ }^{2,4,5}$, we consider Coulomb displacement energies between neighboring IAS one of which is a $T_{z}= \pm T$ ground state. In these cases one has

$$
\Delta E^{\mathrm{C}}(A \alpha T, T)=E^{\mathrm{C}(1)}(A \alpha T)-(6 T-3) E^{\mathrm{C}(2)}(A \alpha T)
$$

for neutron-rich nuclei, and

$$
\Delta E^{\mathrm{C}}(A \alpha T,-T+1)=E^{\mathrm{C}(1)}(A \alpha T)+(6 T-3) E^{\mathrm{C}(2)}(A \alpha T)
$$

for proton-rich nuclei.

We consider separately diagonal regions in the $(N, Z)$ plane, where the valence neutrons and protons are filling the same major shell, and non-diagonal regions,

* With a slight modification of the pairing terms in diagonal shell regions, and without the approximation $\Delta \omega=0$ made there. 
with different major valence shells. As in ref. ${ }^{5}$ ) the magic numbers $N_{i}$ are denoted by $N_{1}=0, N_{2}=2, N_{3}=8, N_{4}=20, N_{5}=28, N_{6}=50, N_{7}=82$ and $N_{8}=126$ for both neutrons and protons. We refer to a shell with $N_{i} \leqslant N \leqslant N_{i+1}$ as an $i$ th major shell and use the notation $(i, j)$ to denote the shell region with the $i$ th neutron and $j$ th proton valence shells. Table 1 shows explicitly for easier reference the definition of the shell regions and some related parameters. The $N_{1}, Z_{1}$ and $A_{1}=N_{1}+Z_{1}$, introduced in eq. (4), denote the corresponding neutron, proton and total nucleon numbers of the doubly magic core of the region. The parameter $\delta$, introduced in eqs. (9)-(12) in non-diagonal regions, denotes the total occupation number for identical nucleons of the lower valence shell of the region. The last column shows the number of experimental C.DE with $\sigma_{\text {cxp }} \leqslant 120 \mathrm{keV}$ in the region (see sect. 4.1.)

\subsection{THE COULOMB ENERGY OF THE GROUND STATE}

As in refs. ${ }^{1-5}$ ) we assume that the ground state Coulomb energy in a given shell region is described phenomenologically by the Carlson-Talmi expression ${ }^{2,3}$ )

$$
\left(\frac{A}{A_{1}}\right)^{\lambda / 3} E^{\mathrm{C}}\left(N_{1}+n, Z_{1}+p\right)=E_{0}+p c+\frac{1}{2} p(p-1) d+\frac{1}{2}\left(p-\delta_{\text {odd } p}\right) \pi
$$

where, as mentioned above, $N_{1}$ and $Z_{1}$ denote the neutron and proton numbers of the doubly magic core, $n$ and $p$ are the respective numbers of valence nucleons, and $\delta_{\text {odd } p}=\frac{1}{2}\left(1-(-1)^{p}\right)$. The coefficient $E_{0}=E^{\mathrm{C}}\left(N_{1}, Z_{1}\right)$ is the Coulomb energy of the core, $c$ is the average Coulomb interaction of a valence proton with the core, $\pi$ is an average Coulomb pairing energy in the major proton valence shell and $d$

TABLE 1

Definition of the shell regions and related parameters

\begin{tabular}{|c|c|c|c|c|c|c|c|}
\hline $\begin{array}{l}\text { Shell } \\
\text { region }\end{array}$ & $N$ & $Z$ & $N_{1}$ & $Z_{1}$ & $A_{1}$ & $\delta$ & $\begin{array}{l}\text { Number of data } \\
\text { total }(n \text {-rich, } p \text {-rich })\end{array}$ \\
\hline$(2,2)$ & $2-8$ & $2-8$ & 2 & 2 & 4 & & $26(17,9)$ \\
\hline$(2,3)$ & $2-8$ & $8-20$ & 2 & 8 & 10 & 6 & $2(0,2)$ \\
\hline$(3,2)$ & $8-20$ & $2-8$ & 8 & 2 & 10 & 6 & $4(4,0)$ \\
\hline$(3,3)$ & $8-20$ & $8-20$ & 8 & 8 & 16 & & $65(39,26)$ \\
\hline$(3,4)$ & $8-20$ & $20-28$ & 8 & 20 & 28 & 12 & $2(0,2)$ \\
\hline$(4,3)$ & $20-28$ & $8-20$ & 20 & 8 & 28 & 12 & $14(14,0)$ \\
\hline$(4,4)$ & $20-28$ & $20-28$ & 20 & 20 & 40 & & $41(36,5)$ \\
\hline$(5,4)$ & $28-50$ & $20-28$ & 28 & 20 & 48 & 8 & $24(24,0)$ \\
\hline$(5,5)$ & $28-50$ & $28-50$ & 28 & 28 & 56 & & $43(42,1)$ \\
\hline$(6,5)$ & $50-82$ & $28-50$ & 50 & 28 & 78 & 22 & $53(53,0)$ \\
\hline$(6,6)$ & $50-82$ & $50-82$ & 50 & 50 & 100 & & $31(31,0)$ \\
\hline$(7,6)$ & $82-126$ & $50-82$ & 82 & 50 & 132 & 32 & $67(67,0)$ \\
\hline$(7,7)$ & $82-126$ & $82-126$ & 82 & 82 & 164 & & $5(5,0)$ \\
\hline$(8,7)$ & $126-184$ & $82-126$ & 126 & 82 & 208 & 44 & $6(6,0)$ \\
\hline$(8,8)$ & $126-184$ & $126-184$ & 126 & 126 & 252 & & \\
\hline
\end{tabular}


is an average orientation-independent interaction between any two valence protons. The factor $\left(A_{1} / A\right)^{\lambda / 3}$ is a scaling factor, to be discussed further in sect. 2.4. The quantities $E_{0}, c$ and $d$ for shell region $(i, i)$ are in ref. ${ }^{5}$ ), respectively, denoted by $\frac{1}{2} Z_{i}\left(Z_{i}-1\right) \omega_{i-1, i-1}, Z_{j} \omega_{i-1, i}$ and $\omega_{i i}$.

Eq. (4) gives ${ }^{3}$ ) the Coulomb energy of a configuration $j^{p}$ of $p$ protons in a single ( $n l j$ ) subshell in a state of lowest seniority $v$ (i.e. $v=0$ for even $p$ and $v=1$ for odd $p$ ). When there are several simultaneously filling valence proton subshells in a well defined state of lowest overall seniority and total proton number $p$, eq. (4) is still expected to be a reasonable approximation provided corresponding interaction parameters in neighboring subshells have numerical values close to each other so that they can be represented by their average in the major shell ${ }^{5,8}$ ). There are also some other special cases ${ }^{9,10}$ ) in which an equation like (4) holds.

However, nuclear ground states have a well defined isospin $T$. This is compatible with having a well defined proton state only in non-diagonal shell regions, and also on the boundaries of diagonal regions, but not in the interior of the latter. Consequently, eq. (4) cannot be valid there.

Hecht ${ }^{11}$ ) [see also ref. ${ }^{7}$ )] calculated the Coulomb energy of a mixed $j^{a}$ configuration of $a=n+p$ nucleons in an $(n l j)$ subshell in a state of given $T$ and lowest seniority $v$ compatible with it. The terms with $E_{0}, c$ and $d$, comprising the main part of $E^{C}$ (see also sect. 4.3.1), are the same as in eq. (4). On the other hand, the expression with the coefficient $\pi$ is more involved, and we did not see how to generalize it to the case of several simultaneously filling mixed subshells.

When the protons are in a well defined state of lowest seniority as on the boundaries of shell regions, and also when there is only one proton or proton-hole in the shell, the exact expressions coincide with eq. (4). On the other hand, in the interior of the shell region the coefficient of $\pi$ is always smaller than that given in eq. (4). The reason for this is that a lowest-seniority state of a mixed $j^{a}$ configuration is a superposition of states of both lowest and higher proton seniorities, and therefore has a lower average number of proton pairs coupled to $J_{\text {pair }}=0$ and hence lower Coulomb pairing. The difference between the two equations increases towards the center of the shell, and stays below $\frac{1}{2} \pi$ for all mixed ground state valence subshells occurring in nature. The maximum values of the difference for the four nuclear parity types are given in appendix A.1.

\subsection{COULOMB DISPLACEMENT ENERGIES IN DIAGONAL REGIONS}

In order to calculate Coulomb displacement energies from eqs. (3a, b) one needs to know $E^{\mathrm{C}(1)}$ and $E^{\mathrm{C}(2)}$. In the present work we use the following expressions:

$$
\begin{aligned}
\left(\frac{A}{A_{1}}\right)^{\lambda / 3} E^{C(0)}- & E_{0}+\frac{1}{2} a c+\left[\frac{1}{8} a(a-2)+\frac{1}{6} T(T+1)\right] d \\
+ & {\left[\frac{a-1}{4}+\delta_{\text {even } a}(-1)^{\frac{1}{2} a-T} \frac{T+1}{12 T}\right] \pi, }
\end{aligned}
$$




$$
\begin{aligned}
& \left(\frac{A}{A_{1}}\right)^{\lambda / 3} E^{C(1)}=\mathrm{c}+\frac{1}{2}(a-1) d+\left[\frac{1}{2}-\delta_{\text {odd } a}(-1)^{\frac{1}{2} a-T} \frac{1}{4 T}\right] \pi, \\
& \left(\frac{A}{A_{1}}\right)^{\lambda / 3} E^{\mathrm{C}(2)}=\frac{1}{6} d+\left[\delta_{\text {even } a}(-1)^{\frac{1}{2} a-T} \frac{1}{12 T^{2}}\right] \pi,
\end{aligned}
$$

where $\delta_{\text {odd } a}=\frac{1}{2}\left(1-(-1)^{a}\right)$, similar to $\delta_{\text {odd } p}$ in eq. (4), and $\delta_{\text {even } a}-\frac{1}{2}\left(1+(-1)^{a}\right)$. Substituing expressions (5)-(7) in eq. (1), one obtains eq. (4) for $T_{z}= \pm T$ ground states.

A derivation of eqs. (5)-(7) is given in appendix A.2.1. Using eqs. (5)-(7) one obtains from eqs. $(3 \mathrm{a}, \mathrm{b})$ :

$$
\begin{aligned}
& \left(\frac{A}{A_{1}}\right)^{\wedge / 3} \Delta E^{\mathrm{C}}\left(Z_{1}+n, Z_{1}+p\right) \\
& \quad=c+p d+\left[\frac{1}{2}+(-1)^{p+1}\left(\delta_{\text {odd } a} \frac{1}{4 T}+\delta_{\text {even } a} \frac{2 T-1}{4 T^{2}}\right)\right] \pi
\end{aligned}
$$

where $n$ and $p$ denote the numbers of valence neutrons and protons of the higher- $T_{z}$ state with the given $\Delta E^{\mathrm{C}}$. For $n$-rich nuclei $T=\frac{1}{2}(n-p)$ and for $p$-rich nuclei $T=\frac{1}{2}(p-n)+1$.

One notes, that the derivation of eq. (8) is based on the assumption that both ground state and IAS are in nuclei within the same shell region. Therefore, it is valid only for nuclei with $Z_{1}+1 \leqslant N \leqslant Z_{2}$ and $Z_{1} \leqslant Z \leqslant Z_{2}-1$.

The coefficients of the large parameters $E_{0}, c$ and $d$ in eqs. (5)-(8) are the same as in eqs. (17)-(20) of ref. $\left.^{5}\right)$, while the coefficient of $\pi$ in eqs. (5), (7) and (8) is different. This is commented on in appendix A.2.

\subsection{COULOMB DISPLACEMENT ENERGIES IN NON-DIAGONAL REGIONS}

We consider a non-diagonal region with valence neutrons and protons in neighboring major shells. For isobaric multiplets having $T_{z}= \pm T$ ground-state members in such regions we use ${ }^{5,12}$ )

$$
\begin{aligned}
\left(\frac{A}{A_{1}}\right)^{\lambda / 3} E^{\mathrm{C}(0)}= & E_{0}+\frac{1}{2}\left(\delta+p_{0}\right) c_{1}+\frac{1}{2} n_{0} c_{2}+\frac{1}{6}\left[\delta(\delta-1)+p_{0}\left(p_{0}-1\right)+p_{0}(\delta-1)\right] d_{1} \\
& +\frac{1}{6} n_{0}\left(n_{0}-1\right) d_{2}+\frac{1}{6} n_{0}\left(p_{0}-\delta\right) I_{12}^{0} \\
& +\frac{1}{6}\left(\delta+2 p_{0}-\delta_{\text {odd } p_{0}}\right) \pi_{1}+\frac{1}{6}\left(n_{0}-\delta_{\text {odd } n_{0}}\right) \pi_{2} \\
\left(\frac{A}{A_{1}}\right)^{\lambda / 3} E^{\mathrm{C}(1)}= & {\left[\left(\delta-p_{0}\right) c_{1}+n_{0} c_{2}+\frac{1}{2}\left[\delta(\delta-1)-p_{0}\left(p_{0}-1\right)\right] d_{1}+\frac{1}{2} n_{0}\left(n_{0}-1\right) d_{2}\right.} \\
& \left.+\frac{1}{2}\left(\delta-p_{0}+\delta_{\text {odd } p_{0}}\right) \pi_{1}+\frac{1}{2}\left(n_{0}-\delta_{\text {odd } n_{0}}\right) \pi_{2}\right] \frac{1}{2 T}
\end{aligned}
$$




$$
\begin{aligned}
\left(\frac{A}{A_{1}}\right)^{\lambda / 3} E^{\mathrm{C}(2)}= & {\left[{ }_{6}^{1}\left(\delta(\delta-1)+p_{0}\left(p_{0}-1\right)-2 p_{0}(\delta-1)\right) d_{1}\right.} \\
& +\frac{1}{6} n_{0}\left(n_{0}-1\right) d_{2}+\frac{1}{3} n_{0}\left(\delta-p_{0}\right) I_{12}^{0} \\
& \left.+\frac{1}{6}\left(\delta-p_{0}-\delta_{\text {odd } p_{0}}\right) \pi_{1}+\frac{1}{6}\left(n_{0}-\delta_{\text {odd } n_{0}}\right) \pi_{2}\right] \frac{1}{2 T(2 T-1)} .
\end{aligned}
$$

Here, $n_{0}$ and $p_{0}$ are the numbers of valence neutrons and protons in their respective shells in the $n$-rich $T_{z}=T$ member of the isomultiplet. The latter comprises a doubly magic core with $Z_{1}$ neutrons and $Z_{1}$ protons, a major shell completely filled with $\delta=Z_{2}-Z_{1}$ neutrons and containing in addition $p_{0}$ valence protons, and another higher major shell with $n_{0}$ valence neutrons. The $\delta_{\text {odd } p_{0}}$ and $\delta_{\text {odd } n_{0}}$ are defined in analogy to $\delta_{\mathrm{odd} p}$ in eq. (4). The coefficients $c_{i}, \pi_{i}$ and $d_{i}(i=1,2)$ are the coefficients $c, \pi$ and $d$ of eq. (4) for the $i$ th major valence shell. The coefficient $I_{12}^{0}$ is the average monopole Coulomb interaction between two protons in major shells 1 and 2 .

Combining eqs. (9)-(11) with eqs. $(3 a, b)$ one obtains:

$$
\begin{aligned}
& \left(\frac{A}{A_{1}}\right)^{\lambda / 3} \Delta E^{C}\left(N_{1}+n, Z_{1}+p\right) \\
& \quad=\left[(\delta-p) c_{1}+n c_{2}+p(\delta-p) d_{1}+n(p-\delta) I_{12}^{0}+\delta_{\text {odd } p} \pi_{1}\right] \frac{1}{2 T},
\end{aligned}
$$

for a $T_{z}=T$ nucleus in a $n$-rich non-diagonal region, and

$$
\begin{aligned}
\left(\frac{A}{A_{1}}\right)^{\lambda / 3} \Delta E^{\mathrm{C}}\left(N_{1}+n, Z_{1}+p\right)= & {\left[(\delta-n+1) c_{1}+(p+1) c_{2}+(\delta-1)(\delta-n+1) d_{1}\right.} \\
& +p(p+1) d_{2}+(p+1)(\delta-n+1) I_{12}^{0} \\
& \left.+(\delta \quad n+1) \pi_{1}+\left(p+1 \quad \delta_{\text {even } p}\right) \pi_{2}\right] \frac{1}{2 T},
\end{aligned}
$$

for a $T_{z}=-T+1$ nucleus in a $p$-rich non-diagonal region. In eqs. $(12 \mathrm{a}, \mathrm{b}) n$ and $p$ refer to the higher- $T_{z}$ state as in eq. (8), $T=\frac{1}{2}(\delta+n-p)$ for $n$-rich nuclei, and $T=\frac{1}{2}(\delta+p-n+2)$ for $p$-rich nuclei. Like eq. (8), eqs. (12a, b) apply only to nuclei with $N_{1}+1 \leqslant N \leqslant N_{2}, Z_{1} \leqslant Z \leqslant Z_{2}-1$.

In ref. ${ }^{5}$ ) eq. (12a) was first obtained by using a particle-hole formalism and then eqs. (9)-(11) were derived using eq. (4) for $T_{z}= \pm T$ nuclei. A derivation starting with eqs. (9)-(11) is given in appendix A.2.2.

Eqs. (9)-(12a) are the same as the corresponding eqs. (27)-(29) and (26) of ref. $\left.{ }^{5}\right)$, with the notation $n_{0}=P, \delta-p_{0}=H$ and $I_{12}^{0}=\omega_{i, i+1}$ for shell region $(i+1, i)$. The corresponding relations for the other coefficients were given after eq. (4).

\subsection{DEPENDENCE OF THE COEFFICIENTS ON MASS NUMBER}

In the present work we scale the coefficients of the Coulomb energy eqs. (4)-(12) by multiplying them by a scaling factor $\left(A_{1} / A\right)^{\wedge / 3}$. As in table $1, A_{1}$ is the mass 
number of the doubly magic core of the region and $\lambda$ is a scaling power. The parameter $\lambda$ is assigned the value $\lambda=1$ for nuclei with valence shells beyond the $1 \mathrm{p}$ shell [from region $(3,3)$ onwards; see table 1 ], and the value $\lambda=0$ for $1 \mathrm{p}$ shell nuclei [region $(2,2)]$. This scaling reflects the gross empirical variation of the nuclear charge radii of stable nuclei $\left.{ }^{13,14}\right)$. Regions $(3,2)$ and $(2,3)$, with both $1 \mathrm{p}$ and $1 \mathrm{~d} 2 \mathrm{~s}$ valence shells, were excluded in the present analysis.

We shall consider scaling further in sect. 4.5 .

\section{Isodiapheric Coulomb displacement energy systematics}

Expressing $\Delta E^{\mathrm{C}}$ in diagonal regions, eq. (8), in terms of $a$ and $T$ one obtains

$$
\begin{aligned}
\left(\frac{A}{A_{1}}\right)^{\lambda / 3} \Delta E^{\mathrm{C}}\left(A_{1}+a, T\right)= & {\left[c-T d+\frac{1}{2} \pi\right]+a\left[\frac{1}{2} d\right] } \\
& +(-1)^{\frac{1}{2} a-T+1}\left[\delta_{\text {odd } a} \frac{1}{4 T}+\delta_{\text {even } a} \frac{2 T-1}{4 T^{2}}\right] \pi
\end{aligned}
$$

for $n$-rich nuclei, and

$$
\begin{aligned}
\left(\frac{A}{A_{1}}\right)^{\lambda / 3} \Delta E^{\mathrm{C}}\left(A_{1}+a, T\right)= & {\left[c+(T-1) d+\frac{1}{2} \pi\right]+a\left[\frac{1}{2} d\right] } \\
& +(-1)^{\frac{1}{2} a+T}\left[\delta_{\text {odd } a} \frac{1}{4 T}+\delta_{\text {even } a} \frac{2 T-1}{4 T^{2}}\right] \pi
\end{aligned}
$$

for $p$-rich nuclei.

For given $T$ and $T_{z}$ in a diagonal region the r.h.s. of eqs. (13a, b) describe sums of a linear function of $a$ with a slope of $\frac{1}{2} d$ which is independent of $T$ and $T_{z}$, and an oscillating function of $a$ with maxima for odd- $p$ nuclei for positive $\pi$. The oscillation amplitude is $(1 / 4 T) \pi$ for half integral values of $T$ and $\left((2 T-1) / 4 T^{2}\right) \pi$ for integral $T$-values, both of which decrease with $T$. The constant term of eqs. $(13 \mathrm{a}, \mathrm{b})$ increases by a constant amount of $\frac{1}{2} d$ when $T_{z}$ decreases by $\frac{1}{2}$.

The multiplication of the equations by the scaling factors $\left(A_{1} / A\right)^{1 / 3}$ has the effect of smoothly reducing the local values of the slope, amplitude of oscillation, and distance between lines of constant $T_{z}$ (isodiapheric lines) when $A$ increases.

When crossing a proton magic number into a higher major shell, the coefficients $\pi$ and $d$, which are defined in terms of average two-body Coulomb matrix elements ${ }^{3}$ ), are expected to decrease discontinuously due to the larger average distance between protons in the higher valence shell. Such a decrease would discontinuously reduce the slope and oscillation amplitude of isodiapheric $\Delta E^{\mathrm{C}}$ lines, and the vertical distance between consecutive lines with different $T_{z}$. Furthermore, one expects a discontinuous downwards shift of the lines themselves, due mainly to the weaker 
interaction $c_{2}-\delta I_{12}^{0}$ of a proton in major shell 2 with the protons in the closed-shell core of region 1 , as compared to $c_{1}^{\star}$.

The expressions of $\Delta E^{C}$ in non-diagonal regions, eq. (12a, b), in terms of $a$ and $T$ are more involved. However, making the rough approximations

$$
d_{1} \approx d_{2} \approx I_{12}^{0}, \quad \pi_{1} \approx \pi_{2}, \quad c_{2} \approx c_{1}+\delta I_{12}^{0}
$$

they become

$$
\left(\frac{A}{A_{1}}\right)^{\lambda / 3} \Delta E^{\mathrm{C}}\left(A_{1}+a, T\right) \approx\left[c-T d+\frac{1}{4 T} \pi\right]+a\left[\frac{1}{2} d\right]+(-1)^{\frac{1}{2} a-T+1} \frac{1}{4 T} \pi
$$

for $n$-rich nuclei, and

$$
\begin{aligned}
\left(\frac{A}{A_{1}}\right)^{\lambda / 3} \Delta E^{\mathrm{C}}\left(A_{1}+a, T\right) \approx & {\left[c+(T-1) d+\left(1-\frac{1}{4 T}\right) \pi\right] } \\
& +a\left[\frac{1}{2} d\right]+(-1)^{\frac{1}{2} a+T} \frac{1}{4 T} \pi
\end{aligned}
$$

for $p$-rich nuclei, where $c$ denotes $c_{1}, d$ denotes the approximate common value of $d_{1}, d_{2}$ and $I_{12}^{0}$, and $\pi$ denotes the approximate common value of $\pi_{1}$ and $\pi_{2}$, eq. (14).

Eqs. $(15 \mathrm{a}, \mathrm{b})$ predict for a non-diagonal region regularities similar to those for a diagonal region considered above, with the difference that the oscillation amplitude is here given by the same expression $(1 / 4 T) \pi$ for both integral and half-integral values of $T$, and the increase of the constant term when $T_{z}$ decreases by $\frac{1}{2}$ ( $T$ decreases to $T-\frac{1}{2}$ in $n$-rich nuclei and $T-\frac{1}{2}$ increases to $T$ in $p$-rich ones) is now $\frac{1}{2} d+(1 / 4 T(2 T-1)) \pi$, which decreases slightly with $T$ as compared to the constant amount of $\frac{1}{2} d$ in a diagonal region.

Fig. 1 shows Coulomb displacement energy lines with constant $T_{z}$ values for $A \leqslant 60$. The lines for $T_{z}=0, \frac{1}{2}$ and 1 are marked in the figure. Lines below that of $T_{z}=T=\frac{1}{2}$ have values of $T=T_{z}$ increasing by $\frac{1}{2}$ from one line to the next one below, and lines above the $T=\frac{1}{2}$ line have values of $T_{z}$ and $T$ decreasing and increasing respectively by $\frac{1}{2}$ from one line to the next one above. Squares and triangles denote data from diagonal and non-diagonal regions, respectively. In each of the three diagonal regions shown one observes a regular system of equidistant and roughly parallel zig-zag lines, with a zig-zag oscillation amplitude mostly decreasing with $T^{\star \star}$.

In the non-diagonal regions the zig-zag oscillations are sometimes less obvious due to lack of data.

The magnitudes of the slope of the lines and of the oscillation amplitude display an overall decrease with $A$, and there are additionally three obvious downward

* More precisely, the downwards discontinuity of the lines at a proton magic number is given by $c_{2}-\delta d_{1}-c_{1}$.

\#* Notable exceptions are the larger amplitude of the $T_{z}=\frac{3}{2}$ line as compared to $T_{z}=1$ in the $1 \mathrm{p}$ shell, and the very small amplitudes of the $T_{z}=\frac{1}{2}$ and $\frac{3}{2}$ lines as compared to $T_{z}=1$ and 2 in the $1 f_{7 / 2}$ shell. 


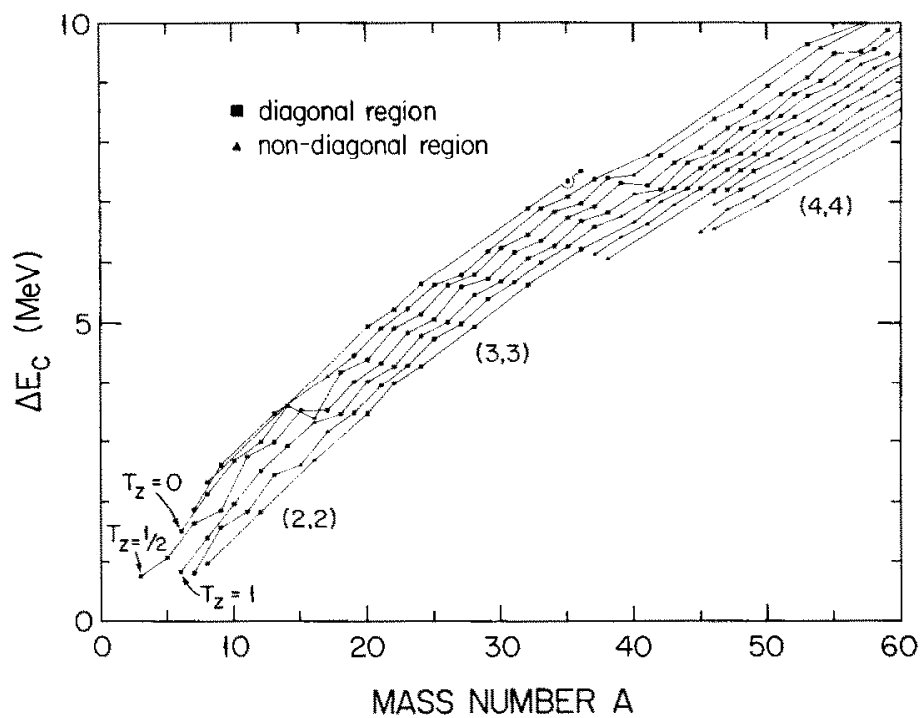

Fig. 1. Coulomb displacement energy systematics for $A \leqslant 60$. Data points for isodiapheres (nuclei with the same neutron excess) are connected by straight lines. Data are from refs. ${ }^{15.16}$ ) and from table A.2.

discontinuities of the $T_{z}=\frac{1}{2}$ line between the $1 \mathrm{p}, 1 \mathrm{~d} 2 \mathrm{~s}, 1 \mathrm{f}_{7 / 2}$ and $1 \mathrm{f}_{5 / 2} 2 \mathrm{p}_{1 g_{9 / 2}}$ shell regions. Similar discontinuities are observable in the $T_{z}=0$ and 1 lines.

These regularities are all in agreement with eqs. $(13 a, b)$ and $(15 a, b)$.

Fig. 2 shows the entire systematics of isodiapheric Coulomb displacement energy lines, where the data with $A \geqslant 16$ are multiplied by $\left(\frac{1}{16} A\right)^{1 / 3}$. Here the zig-zag oscillations are in each shell region superimposed on a system of parallel equidistant straight lines. The slopes of the lines in different regions are about the samc, indicating a mainly $A^{-1 / 3}$ variation of the average Coulomb interactions beyond $A=16^{\star}$.

The high linearity with respect to $A$ suggests the usefulness of this systematics when plotted on a larger scale (not shown here), both for pointing out possibly inaccurate or otherwise conspicuous data, and for predictions. As a rule, neighboring lines do not intersect ${ }^{\star \star}$. Data points where the distance between lines is significantly smaller than indicated by the systematics are for the proton-rich nuclei ${ }^{16} \mathrm{O}(T=1)$, ${ }^{7} \mathrm{Be}\left(T=\frac{3}{2}\right),{ }^{8} B(T=2)$ and ${ }^{35} \mathrm{~K}\left(T=\frac{5}{2}\right)$, whose values seem to be too low, and for the neutron-rich nuclei ${ }^{71} \mathrm{Ga}\left(T=\frac{9}{2}\right.$, too low $),{ }^{140} \mathrm{Ce}(T=12$, too high $),{ }^{139} \mathrm{La}\left(T=\frac{25}{2}\right.$, too low) and ${ }^{165} \mathrm{Ho}\left(T=\frac{31}{2}\right.$, too high). These points, as well as ${ }^{5} \mathrm{He}\left(T=\frac{1}{2}\right.$, too high $)$ and ${ }^{193} \mathrm{Os}\left(T=\frac{41}{2}\right.$, too high) which are not conspicuous in the systematics due to scarcity of nearby data, also come out with significantly large deviations in the

* Another indication for an $A^{-1 / 3}$ dependence is the fact that points belonging to the same element are often lying on a horizontal line in heavier nuclei with higher $T$-values, where the oscillating pairing terms are negligible. See also fig. 2 of ref. ${ }^{16}$ ).

** Except for lines in the light proton-rich nuclei, and also at ${ }^{139} \mathrm{La}$, where this is probably due to a large experimental uncertainty. 


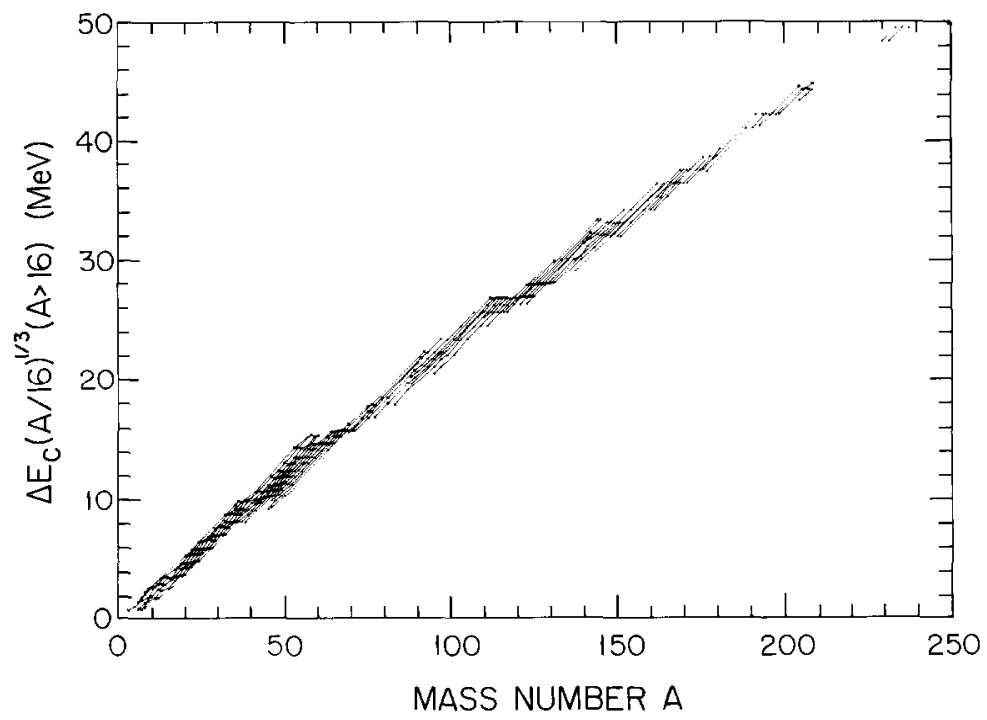

Fig. 2. Same as fig. 1 for $A \leqslant 238$. Data for $A \geqslant 16$ are multiplied by $\left(\frac{1}{16} A\right)^{1 / 3}$.

least-squares adjustments described in sect. 4.2. The high value of ${ }^{5} \mathrm{He}$ and low values of the proton-rich nuclei are presumably mainly due to particle instabilities and Thomas-Ehrman shifts. The values of ${ }^{140} \mathrm{Ce},{ }^{139} \mathrm{La}$ and ${ }^{165} \mathrm{Ho}$ agree with the systematics within their respective experimental uncertainties of 106, 120 and $106 \mathrm{keV}$.

The relation of the systematics to the liquid drop model is considered in sect. 5.1.

\section{Adjustment of the equations to the data}

\subsection{THE EXPERIMENTAL DATA}

We used as data base 383 Coulomb displacement energies as compared to 288 $n$-rich values used earlier in ref. ${ }^{5}$ ). (Data with $Z>N$ were not used in the data base of these calculations.) The data were taken from two recent compilations ${ }^{15,16}$ ), and from more recent literature. These additional energies are given in appendix A.3.

We used only data with experimental uncertainties not exceeding $120 \mathrm{keV}$, which is about twice the resulting final statistical error. The nuclei thus excluded and their experimental uncertainties in $\mathrm{keV}$ are: ${ }^{53} \mathrm{Co}(162),{ }^{57} \mathrm{Cu}(144),{ }^{75} \mathrm{As}(150),{ }^{103} \mathrm{Rh}$ (150), ${ }^{133} \mathrm{Ba}(146),{ }^{141} \mathrm{Pr}(250)$ and ${ }^{159} \mathrm{~Tb}(150)$.

In 19 out of the above cases, where no ground state data were available, we used excited states with $E_{\mathrm{x}}<500 \mathrm{keV}$ as in ref. ${ }^{5}$ ). We noticed that in the 81 cases in ref. ${ }^{16}$ ) where both ground state and 1 st excited state data up to $500 \mathrm{keV}$ are available, they do not differ by more than $90 \mathrm{keV}$. 


\subsection{LEAST-SQUARES ADJUSTMENTS}

4.2.1. Coulomb displacement energy eqs. (8) and $(12 a, b)$. We performed leastsquares adjustments of eqs. (8) and $(12 a, b)$ to the data, with equal weights as was done in refs. ${ }^{1,5}$ ). First we adjusted eqs. (8) and (12a) separately in individual shell regions [eq. (12b) could not be adjusted in single proton-rich non-diagonal regions, as there are only two data points more accurate than $120 \mathrm{keV}$ in each of regions $(2,3)$ and $(3,4)$ and none in the others]. As mentioned above, we used a scaling power $\lambda=1$ from region $(3,3)$ onwards and $\lambda=0$ in region $(2,2)$.

Then we performed separate adjustments in combined groups of four (only for $i=3$ ) or three (for $i \geqslant 4$ ) regions comprising two neighboring diagonal regions $(i, i)$ and $(i+1, i+1)$ and the non-diagonal regions $(i+1, i)$ and $(i, i+1)$ between them. These adjustments were performed from region $(3,3)$ onwards with the scaling power $\lambda=1$.

Finally, we performed one big least-squares adjustment to all data from region $(3,3)$ onwards with $\lambda=1$.

We noticed, that within the resulting statistical errors ${ }^{\star}$ a given parameter has the same adjusted value in the last two procedures of combined calculations, and also in the calculation in an individual diagonal region ${ }^{\star}$. On the other hand, the value(s) obtained in adjustment(s) of individual non-diagonal regions often did not agree with the other values.

We also noticed, that the resulting statistical errors of the parameters were often smaller for adjustments in combined regions than in individual regions.

Table 2 shows the values of the coefficients resulting from the simultaneous adjustment to all data from region $(3,3)$ onwards, and also the adjusted coefficients for region $(2,2)$. For convenience the coefficients are uniformly rounded off to $10^{-3} \mathrm{keV}$, in order that ground state Coulomb energies calculated from them will have rounding-off errors less than $1 \mathrm{keV}$ in all practical cases. The statistical errors of the coefficients are rounded off to two significant figures. The last column of the table shows for each region the corresponding scaling parameters $A_{1}$ and $\lambda$, to be used with eqs. (4)-(12a, b). The $A_{1}$ values in part (a) are regional values from table 1. For convenience in calculations we also give upscaled values of the coefficients as in part (b), to be used with the same value $A_{1}=16$ in all shell regions.

The table shows the adjusted values of $c+\frac{1}{2} \pi$ rather than $c$, except for region $(8,8)$. We adjusted $c+\frac{1}{2} \pi$ and $\pi$ rather than $c$ and $\pi$, since $c$ and $\pi$ could not be separately determined from the data in regions $(6,6)$ and $(7,7)$ whereas the combination $c+\frac{1}{2} \pi$ could as explained below (see also remark $a$ in table 2).

The statistical errors of the coefficients $d_{7}, I_{87}^{0}$ and in particular $c_{8}$ are considerably higher than in lower shell regions. Practical implications of this are considered in

* The statistical error or standard deviation of the $i$ th parameter is given by the product of the standard deviation, eq. (28), and the square root of the $i$ th diagonal element of the inverse matrix of the least-squares normal equations.

** Except for the parameters of regions $(7,7)$ and $(8,7)$ which have very few experimental data. 
TABLE 2

Adjusted values of Coulomb interaction parameters for eqs. (8) and (12a, b)

\begin{tabular}{|c|c|c|c|c|c|c|}
\hline \multirow{2}{*}{$\begin{array}{c}\text { Region } \\
\quad(i, i)\end{array}$} & \multicolumn{4}{|c|}{ Interaction parameters $(\mathrm{keV})$} & \multicolumn{2}{|c|}{$\begin{array}{c}\text { Scaling } \\
\text { parameters }\end{array}$} \\
\hline & $c_{i}+\frac{1}{2} \pi_{i}$ & $d_{i}$ & $\pi_{i}$ & $I_{i+1, i}^{0}$ & $A_{1}$ & $\lambda$ \\
\hline \multicolumn{7}{|c|}{ (a) Regional scaling } \\
\hline$(2,2)$ & $966.832 \pm 41.0$ & $510.721 \pm 15.0$ & $173.158 \pm 75.0$ & - & 4 & 0 \\
\hline$(3,3)$ & $3749.369+13.0$ & $547.009 \pm 2.2$ & $189.512 \pm 24.0$ & $434.149+21.0$ & 16 & 1 \\
\hline$(4,4)$ & $7481.228 \pm 12.0$ & $422.861 \pm 3.7$ & $92.557 \pm 26.0$ & $361.552 \pm 14.0$ & 40 & 1 \\
\hline$(5,5)$ & $9657.735+11.0$ & $361.355 \pm 1.8$ & $81.791 \pm 35.0$ & $394.057 \pm 5.7$ & 56 & 1 \\
\hline$(6,6)$ & $14569.655 \pm 9.2$ & $296.886 \pm 2.0$ & - & $314.749 \pm 2.9$ & 100 & 1 \\
\hline$(7,7)$ & $20459.799 \pm 16.0$ & $213.430 \pm 34.0$ & - & $353.641 \pm 78.0$ & 164 & 1 \\
\hline$(8,8)$ & $\left.30359.240 \pm 2400.0^{\mathrm{a}}\right)$ & - & - & - & 252 & 1 \\
\hline \multicolumn{7}{|c|}{ (b) Global scaling } \\
\hline$(2,2)$ & $966.832 \pm 41.0$ & $510.721 \pm 15.0$ & $173.158 \pm 75.0$ & - & 16 & 0 \\
\hline$(3,3)$ & $3749.369 \pm 13.0$ & $547.009 \pm 2.2$ & $189.512 \pm 24.0$ & $434.149 \pm 21.0$ & 16 & 1 \\
\hline$(4,4)$ & $10153.589 \pm 16.0$ & $573.910 \perp 5.0$ & $125.619 \pm 35.0$ & $490.702 \pm 19.0$ & 16 & 1 \\
\hline$(5,5)$ & $14663.286 \pm 16.0$ & $548.643 \pm 2.7$ & $124.183 \pm 53.0$ & $598.294 \pm 8.7$ & 16 & 1 \\
\hline$(6,6)$ & $26837.534 \pm 17.0$ & $546.868 \pm 3.7$ & - & $579.773 \pm 5.4$ & 16 & 1 \\
\hline$(7,7)$ & $44443.608+35.0$ & $463.622+74.0$ & - & $768.194+170.0$ & 16 & 1 \\
\hline$(8,8)$ & $\left.76099.958 \pm 6000.0^{\mathrm{a}}\right)$ & - & - & - & 16 & 1 \\
\hline
\end{tabular}

a) This is the value for the parameter $c_{8}$. It was obtained from the data in regions $(7,7)$ and $(8,7)$ where the parameter $\pi_{8}$ does not appear in the adjusted eq. (12a).

sect. 4.4. Like the disagreements in the results for these regions, mentioned in the last footnote, these higher statistical errors are presumably due to the paucity of experimental data in regions $(7,7)$ and $(8,7)$.

The coefficients $I_{32}^{0}, \pi_{6}, \pi_{7}, d_{8}, \pi_{8}$ and $I_{98}^{0}$ are missing in the table. The $I_{32}^{0}$ belongs to regions $(3,2)$ and $(2,3)$, which were left out from the adjustments, and the last three can be determined only from data in regions $(7,8)$ and $(8,8)$ which are presently beyond experimental reach. The other two coefficients, $\pi_{6}$ and $\pi_{7}$, were held fixed on the value 0 , since otherwise $\pi_{6}$ comes out statistically insignificant (by which one means having a statistical error larger than or about equal to its value), and the statistically significant adjusted value of $\pi_{7}$ comes out negative, which is physically meaningless [eq. (24)]. Like the other irregularities mentioned above, the last one is presumably due to the paucity of data determining $\pi_{7}$ [mainly the odd $Z$ nucleus ${ }^{209} \mathrm{Bi}$ in region $(7,7) 1$.

4.2.2. The Carlson-Talmi Coulomb displacement energy equation. For the sake of comparison, we made as well least-squares adjustments of the Carlson-Talmi Coulomb displacement energy equation ${ }^{2,3}$ )

$$
\left(\frac{A}{A_{1}}\right)^{\lambda / 3} \Delta E^{C}\left(N_{1}+n, Z_{1}+p\right)=c+p d+\delta_{\text {odd } p} \pi
$$


both in individual regions, and in combined groups of two regions $(i, i)$ and $(i+1, i)$ with $i \geqslant 3$. We used the same scaling factors as before. As in the case of eqs. (8) and $(12 a, b)$, the values obtained for a given parameter in a combined calculation and in an individual diagonal region come out the same within their statistical errors, and the statistical errors are somewhat smaller in combined calculations.

On the other hand, as compared to eqs. (8) and (12a, b), the coefficient $\pi_{6}$ is not insignificant anymore, and the uncertainty of $d_{7}$ is reduced to the same order of magnitude as for lighter shell regions. The last point is considered further in sect. 4.4.

The values of the coefficients obtained in the combined adjustments and also those of region $(2,2)$ are given in table 3 organized similarly to table 2.

\subsection{DISCUSSION OF THE RESULTS}

4.3.1. Signs and magnitude relationships of the coefficients. We discuss first some qualitative features of the adjusted values, using the shell-model expressions for single subshells as a guideline. One observes that none of the coefficients in tables 2 and 3 is negative, which is also obvious from the systematics presented in figs. 1 and 2. This is due to the repulsive nature of the Coulomb interaction between the protons. As a matter of fact, for single $(n l j)$ subshells the coefficients are given by

$$
\begin{aligned}
c_{j} & =\sum_{\alpha}\left(2 j_{\alpha}+1\right) I_{j_{\alpha} j}^{0}, \\
d_{j} & =F^{0}(n l j, n l j)-\left(\frac{2}{2 j-1}\right)\left(\sum_{k \text { even } \neq 0} F^{k}(n l j, n l j) \frac{\left\langle j\left\|C^{(k)}\right\| j\right\rangle^{2}}{2 j+1}\right), \\
\pi_{j} & =\left(\frac{2 j+1}{2 j-1}\right)\left(\sum_{k \text { crcn } \rightarrow 0} F^{k}(n l j, n l j) \frac{\left\langle j\left\|C^{(k)}\right\| j\right\rangle^{2}}{2 j+1}\right), \\
I_{j j^{\prime}}^{0} & =F^{0}\left(n l j, n^{\prime} l^{\prime} j^{\prime}\right)-\sum_{k} G^{k}\left(n l j, n^{\prime} l^{\prime} j^{\prime}\right) \frac{\left\langle j\left\|C^{(k)}\right\| j^{\prime}\right\rangle^{2}}{(2 j+1)\left(2 j^{\prime}+1\right)},
\end{aligned}
$$

\begin{tabular}{|c|c|c|c|c|c|}
\hline \multirow{2}{*}{$\begin{array}{c}\text { Region } \\
(i, i)\end{array}$} & \multicolumn{3}{|c|}{ Interaction parameters $(\mathrm{keV})$} & \multicolumn{2}{|c|}{$\begin{array}{c}\text { Scaling } \\
\text { parameters }\end{array}$} \\
\hline & $c_{i}+\frac{1}{2} \pi_{i}$ & $d_{i}$ & $\pi_{i}$ & $A_{1}$ & $\lambda$ \\
\hline$(2,2)$ & $971.454 \pm 42.0$ & $508.692 \pm 15.0$ & $107.754 \pm 47.0$ & 4 & 0 \\
\hline$(3,3)$ & $3755.130 \pm 16.0$ & $545.236 \pm 2.4$ & $90.052 \pm 17.0$ & 16 & 1 \\
\hline$(4,4)$ & $7494.983 \pm 14.0$ & $419.550 \pm 3.5$ & $41.120+16.0$ & 40 & 1 \\
\hline$(5,5)$ & $9653.394 \pm 10.0$ & $359.748 \pm 0.82$ & $42.681 \pm 13.0$ & 56 & 1 \\
\hline$(6,6)$ & $14569.923 \pm 16.0$ & $294.937 \pm 0.74$ & $40.592 \pm 26.0$ & 100 & 1 \\
\hline$(7,7)$ & $20399.262 \pm 17.0$ & $231.546 \pm 2.7$ & - & 164 & 1 \\
\hline
\end{tabular}

TABLE 3

Adjusted values of Coulomb interaction parameters for eq. (16) 
where $F^{k}$ and $G^{k}$ are the direct and exchange radial Slater integrals for the Coulomb interaction, and $C_{q}^{(k)}$ are normalized spherical harmonics. The summation in eq. (17) runs over all closed proton $\left(n_{\alpha} l_{\alpha} j_{\alpha}\right)$ subshells of the core.

For given subshells the $F^{(k)}$ are positive decreasing functions of $k$. The $G^{k}$ are also positive, and for different $(n l j)$ and $\left(n^{\prime} l^{\prime} j^{\prime}\right)$ subshells they are smaller than the corresponding $F^{k}$. The coefficients of the radial parameters are given by ${ }^{3}$ )

$$
\begin{gathered}
\frac{\left\langle j\left\|C^{(k)}\right\| j\right\rangle^{2}}{2 j+1}=\left(\begin{array}{ccc}
j & k & j \\
-\frac{1}{2} & 0 & \frac{1}{2}
\end{array}\right)^{2}(2 j+1) \\
=\mathrm{O}\left[\max \left(\frac{1}{2 j+1}, \frac{1}{2 k+1}\right)\right], \\
\frac{\left\langle j_{1}\left\|C^{(k)}\right\| j_{2}\right\rangle^{2}}{\left(2 j_{1}+1\right)\left(2 j_{2}+1\right)}=\left(\begin{array}{ccc}
j_{1} & k & j_{2} \\
-\frac{1}{2} & 0 & \frac{1}{2}
\end{array}\right)^{2} \\
=\mathrm{O}\left[\max \left(\frac{1}{\left(2 j_{1}+1\right)\left(2 j_{2}+1\right)}, \frac{1}{\left(2 j_{1}+1\right)(2 k+1)}, \frac{1}{(2 k+1)\left(2 j_{2}+1\right)}\right)\right],
\end{gathered}
$$

which are smaller than 1 [the order of magnitude estimates in eqs. (21) and (22) result from the normalization of the $3 j$ symbols]. Thus, all four of the coefficients defined by eqs. (17)-(20), as well as the coefficient

$$
E_{0}=\sum_{(\alpha \beta)}\left(2 j_{\alpha}+1\right)\left(2 j_{\beta}+1\right) I_{j_{\alpha} j_{\beta}}^{0}+\sum_{\alpha}\left(j_{\alpha}\left(2 j_{\alpha}+1\right) d_{\alpha}+\frac{1}{2}\left(2 j_{\alpha}+1\right) \pi_{\alpha}\right),
$$

where the summation extends over all pairs of closed proton subshells of the core, are expected to be positive, and the same presumably applies to the corresponding average interactions used in the present work.

We consider now the relative magnitudes of the coefficients. Due to the summation appearing in eqs. (17) and (23) one expects to have the relations

$$
E_{0}>c>\pi, d, I_{12}^{0}>0
$$

in all cases where there are several subshells in the core. The relationships (24) are indeed satisfied by the coefficients in tables 2 and 3 and the coefficient $E_{0}$ from table 8 below in all shell regions from $(3,3)$ onwards.

Consider next the three valence subshell coefficients $\pi, d$ and $I_{12}^{0}$. From eqs. (18)-(22) one expects that the intrasubshell coefficients obey the relation

$$
d>\pi>0
$$

which is indeed satisfied by the coefficients in tables 2 and 3 . However, it is not obvious a priori from the above considerations what to expect for the relative magnitude of $I_{12}^{0}$ and $d$, as one presumably has both $F^{0}(n l j, n l j)>F^{0}\left(n l j, n^{\prime} l^{\prime} j^{\prime}\right)$ and $G^{k}\left(n l j, n^{\prime} l^{\prime} j^{\prime}\right)<F^{k}(n l j, n l j)$. The differences $I_{12}^{0}-d_{1}$ are in fact negative for regions $(3,3)$ and $(4,4)$ and positive for regions $(5,5),(6,6)$ and $(7,7)$. 
Relations (24) and (25) are satisfied as well by the corresponding earlier values of the coefficients of ref. ${ }^{5}$ ).

Eq. (25) is in sharp contrast to the nuclear case, where the $d$ parameters are positive and have typically magnitudes of a few hundred $\mathrm{keV}$, whereas the pairing energies are negative, with typical magnitudes of $1-2 \mathrm{MeV}$. Altogether, instead of eq. (25) one has $-\pi^{\text {nuclear }}>d^{\text {nuclear }}>0$. This different behavior is presumably mainly due to the fact that for attractive short-range nuclear interactions the radial integrals are negative, and the magnitudes of $F^{k}$ for given subshells increase with $k$. (For $V_{12}=\delta\left(\boldsymbol{r}_{1}-\boldsymbol{r}_{2}\right)$ one has $F^{k}=G^{k}=(2 k+1) F^{0}$.) In addition, there are non-central and spin-dependent nuclear forces, for which eqs. (18) and (19) are not valid.

We finally consider the shell dependence of the coefficients. Due to the summations appearing in eqs. (17) and (23) it is obvious that both $E_{0}$ and $c$ must increase from lighter to heavier nuclei and $E_{0}$ would increase faster. On the other hand, due to the larger avcrage distancc between the protons in the higher shells in heavier nuclei, the coefficients $\pi, d$ and $I_{12}^{0}$ are expected to be smaller in heavier nuclei. These expectations for the coefficients $E_{0}, c, \pi$ and $d$ are born out by the systematics in fig. 1 and by the coefficients in parts (a) of tables 2 and $8^{\star}$ and in table 3 . On the other hand, the coefficients $I_{i+1, i}^{0}$ are sometimes larger in higher shells.

In part (b) of table 2 the coefficients $d$ in the various shells have similar magnitudes [except for region $(7,7)$ ]. This is in agreement with the mainly $A^{-1 / 3}$ dependence of these coefficients discussed in connection with fig. 2 in sect. 3 . The smaller value of $d_{7}$ is discussed further in sects. 4.4 and 5.3.

4.3.2. Agreement with the data. Useful statistical parameters for discussing the agreement of the theory with part or all of the data are the mean of the deviations $\varepsilon$ and the root mean square (r.m.s.) deviation $\sigma_{\text {r.m.s. }}$ defined by

$$
\begin{aligned}
\varepsilon & =\frac{\sum\left(\Delta E_{\mathrm{calc}}^{\mathrm{C}}-\Delta E_{\mathrm{exp}}^{\mathrm{C}}\right)}{N}, \\
\sigma_{\text {r.m.s. }} & =\left[\frac{\sum\left(\Delta E_{\mathrm{calc}}^{\mathrm{C}}-\Delta E_{\mathrm{exp}}^{\mathrm{C}}\right)^{2}}{\mathrm{~N}}\right]^{1 / 2},
\end{aligned}
$$

where $N$ is the number of data considered. The overall goodness of fit is traditionally expressed in terms of the standard deviation $\sigma$, defined as

$$
\sigma=\left[\frac{\sum\left(\Delta E_{\mathrm{calc}}^{\mathrm{C}}-\Delta E_{\mathrm{exp}}^{\mathrm{C}}\right)^{2}}{N-m}\right]^{1 / 2},
$$

where $N$ is the total number of data participating in the least-squares adjustment and $m$ is the number of adjustable parameters.

* Except on the transition from region $(2,2)$ to $(3,3)$, where $d_{2}<d_{3}$. However, this does not contradict the above reasoning, as nuclear radii are about the same for the $1 \mathrm{p}$ shell and the beginning of the $1 \mathrm{~d} 2 \mathrm{~s}$ shell. 
The standard deviations for eqs. (8) and (12a, b) with the scaling discussed above are $113 \mathrm{keV}$ for region $(2,2)$ and $49 \mathrm{keV}$ for the combined calculation of all shell regions beyond $(3,3)$. This gives a total standard deviation of $55 \mathrm{keV}$ (table 5). The corresponding numbers for the Carlson-Talmi equation (16) are 113, 56 and $62 \mathrm{keV}$. These results will be considered further in sects. 4.4 and 4.5 .

In order to gain better information on the distribution of the deviations, we calculated for each shell region, like in ref. ${ }^{5}$ ), the mean $\varepsilon$ and the r.m.s. deviation $\sigma_{\text {r.m.s. }}$ for the parameter values of tables 2 and 3 . The results are presented in table 4 .

We discuss first mean values for eqs. (8) and (12a,b). One obscrves that $\varepsilon$ usually has magnitudes of a few $\mathrm{keV}$, except for the proton-rich region $(3,4)$, where $\varepsilon=76 \mathrm{keV}$, and region $(7,7)$ with 5 data points, where $\varepsilon=45 \mathrm{keV}$. Moreover, in a combined calculation of all data with $\lambda=0.80$ (see table 5) the resulting value of $\varepsilon$ for region $(2,3)$ is $185 \mathrm{keV}$. These findings for regions $(2,3)$ and $(3,4)$ are consistent with the occurrence of conspicuously low $\Delta E^{\mathrm{C}}$ values in the systematics in sect. 3 . They are also in agreement with results obtained for these regions by extrapolation in ref. ${ }^{5}$ ) (table 3 there).

A similar higher value of $\varepsilon$ in region $(3,4)$ occurs for eq. (16) as well.

One observes further, that the $\sigma_{\text {r.m.s. }}$ values for eqs. (8) and $(12 \mathrm{a}, \mathrm{b})$ are on the whole larger in diagonal than in non-diagonal regions. This is probably related to the approximate nature of the Carlson-Talmi eq. (4) in diagonal regions. We

TABLE 4

Values of $E$ and $\sigma_{\text {r.m.s. }}$ (in keV) for eqs. (8) $+(12 \mathrm{a}, \mathrm{b})$ and (16)

\begin{tabular}{|c|c|c|c|c|c|}
\hline \multirow{2}{*}{ Region } & \multirow{2}{*}{$\begin{array}{c}\text { No. of data } \\
N\end{array}$} & \multicolumn{2}{|c|}{ Eqs. $(8)$ and $(12 a, b)$} & \multicolumn{2}{|c|}{ Eq. (16) } \\
\hline & & $\varepsilon$ & $\sigma_{r . m . s .}$ & $\varepsilon$ & $\sigma_{\text {r.m.s }}$ \\
\hline \multicolumn{6}{|l|}{ (a) Diagonal regions } \\
\hline$(i, i):(2,2)$ & 26 & 0.0 & 106.1 & 0.0 & 106.1 \\
\hline$(3,3)$ & 65 & -4.2 & 52.8 & -6.7 & 56.6 \\
\hline$(4,4)$ & 41 & 0.1 & 65.3 & 4.7 & 67.3 \\
\hline$(5,5)$ & 43 & 3.6 & 49.6 & -9.4 & 52.3 \\
\hline$(6,6)$ & 31 & -4.1 & 44.9 & -24.5 & 54.1 \\
\hline$(7,7)$ & 5 & 45.0 & 59.2 & -7.7 & 41.8 \\
\hline total diag. $i \geqslant 3$ & 185 & -0.1 & 54.1 & -7.8 & 57.5 \\
\hline \multicolumn{6}{|l|}{ (b) Non-diagonal regions } \\
\hline$(i, j):(4,3)$ & 14 & 9.4 & 71.4 & 27.0 & 74.6 \\
\hline$(3,4)$ & 2 & 75.6 & 76.1 & 57.3 & 63.2 \\
\hline$(5,4)$ & 24 & 5.7 & 32.8 & 9.4 & 33.4 \\
\hline$(6,5)$ & 53 & -1.2 & 31.3 & 7.9 & 49.2 \\
\hline$(7,6)$ & 67 & -3.2 & 33.9 & 11.4 & 57.1 \\
\hline$(8,7)$ & 6 & 9.2 & 32.4 & 6.5 & 25.0 \\
\hline total non-diag. $i, j \geqslant 3$ & 166 & -0.5 & 38.3 & 8.9 & 53.3 \\
\hline total $i, j \geqslant 3$ & 351 & -0.3 & 47.3 & 0.1 & 55.5 \\
\hline total & 377 & -0.3 & 53.5 & 0.1 & 60.4 \\
\hline
\end{tabular}


TABLE 5

Dependence of the standard deviations (in $\mathrm{keV}$ ) on the scaling factors for eqs. (8) and $(12 \mathrm{a}, \mathrm{b})$

\begin{tabular}{ccrr}
\hline Region & $\left.(N, m)^{\mathrm{a}}\right)$ & $\left.\sigma\left(\lambda_{2}\right)^{\mathrm{b}}\right)$ & $\sigma\left(\lambda_{\text {hest }}\right)$ \\
\hline$(2,2)$ & $(26,3)$ & $112.8(0)$ & $112.1(0.10)$ \\
$(3,3)$ & $(65,3)$ & $53.7(1)$ & $49.2(0.85)$ \\
$(4,4)$ & $(41,3)$ & $67.7(1)$ & $43.6(0.50)$ \\
$(5,5)$ & $(43,3)$ & $50.5(1)$ & $50.5(1.00)$ \\
$(6,6)$ & $(31,2)$ & $45.7(1)$ & $41.9(0.85)$ \\
$(7,7)$ & $(5,2)$ & $47.7(1)$ & $47.7(1.00)$ \\
& & & \\
$(2,2)+(3,2)+(2,3)+(3,3)$ & $(97,7)$ & - & $92.0(0.65)$ \\
$(3,3)+(4,3)+(3,4)+(4,4)$ & $(122,7)$ & $61.7(1)$ & $52.1(0.70)$ \\
$(4,4)+(5,4)+(5,5)$ & $(108,7)$ & $54.9(1)$ & $51.5(0.85)$ \\
$(5,5)+(6,5)+(6,6)$ & $(127,6)$ & $42.4(1)$ & $42.0(0.95)$ \\
$(6,6)+(7,6)+(7,7)$ & $(103,5)$ & $39.2(1)$ & $37.8(0.90)$ \\
$(7,7)+(8,7)$ & $(11,4)$ & $37.0(1)$ & $37.0(1.00)$ \\
total $i, j \geqslant 3,3$ & $(351,19)$ & $48.6(1)$ & $46.1(0.85)$ \\
total $i, j \geqslant 2,2$ & $(383,23)$ & $\left.65.2(\lambda=1)^{\mathrm{c}}\right)$ & $61.4(0.80)$ \\
total $(2,2)+(i, j \geqslant 3,3)$ & $(377,22)$ & 55.1()$\left.^{\mathrm{d}}\right)$ & 52.9()$\left.^{\mathrm{e}}\right)$ \\
\hline
\end{tabular}

a) $N$ and $m$ denote the respective numbers of data and adjustable parameters [eq. (28)].

b) $\lambda_{2}$ denotes the scaling power 0 for region $(2,2)$ and 1 for $(i, j)$ with $i, j \geqslant 3$ (table 2).

c) This is not $\lambda_{2}$ from table 2 .

d) This is the total $\sigma$ for $\lambda(2,2)=0$ and $\lambda(i, j \geqslant 3,3)=1$.

c) This is the total $\sigma$ for $\lambda(2,2)=0.10$ and $\lambda(i, j \geqslant 3,3)=0.85$.

mentioned in sect. 2.1 that this approximation introduces errors of the order of fractions of $\pi$. The differences between the $\sigma_{\text {r.m.s. }}$ values in diagonal and in nondiagonal regions are of this order of magnitude.

On the other hand, the difference between the $\sigma_{\text {r.m.s. }}$ values in diagonal and in non-diagonal regions is less conspicuous for eq. (16). This is discussed in sect. 4.4.

Finally, one observes that as in ref. ${ }^{5}$ ) the $\sigma_{\text {r.m.s. }}$ values in the $1 \mathrm{p}$ shell are considerably higher than in heavier nuclei for both equations. This reflects a higher degree of smoothness of the Coulomb energy as function of nucleon numbers beyond the $1 \mathrm{p}$ shell, and is presumably related to the weaker average Coulomb interaction in heavier larger nuclei. The situation for nuclear mass equations is similar.

\subsection{COMPARISON OF EQS. (8) AND (12a, b) WITH THE}

\section{CARLSON-TALMI EQUATION}

One observes that within the statistical errors the numerical values of the coefficients $c+\frac{1}{2} \pi$ and $d$ in part (a) of table 2 and in table 3 , obtained respectively from eqs. (8) and (12a, b) and from eq. (16), are the same except for $c_{7}+\frac{1}{2} \pi_{7}$. The 
latter value of table 3 is determined from the small number of the data in regions $(7,7)$ and $(7,8)$. However, the pairing energies $\pi$ are larger in table 2 .

These results are expected, as the terms with $c+\frac{1}{2} \pi$ and $d$ are the same in diagonal regions for both equations, and the pairing terms with $\pi$ are small. On the other hand, since the coefficient of $\pi$ is independent of $T$ in eq. (16), but decreases when $T$ increases in eq. (8), the value of $\pi$ resulting from the adjustments to the data should be larger for the latter.

The r.m.s. deviations in table 4 have similar magnitudes for the two equations in diagonal regions whereas the r.m.s. deviations in non-diagonal rcgions shown in table 4 are mostly smaller for eqs. (12a, b) than for eq. (16), as mentioned earlier in sect. 4.3.2. The overall $\sigma_{\text {r.m.s. }}$ values for diagonal regions from $(3,3)$ onwards for eqs. (12a, b) and (16) are 54 and $57 \mathrm{keV}$, respectively, and for non-diagonal regions they are 38 and $53 \mathrm{keV}$.

Thus, it seems that in non-diagonal regions, eq. (12a,b) with its $T$-dependence and its additional parameter $I_{12}^{0}$ is in better agreement with the data than eq. (16).

It must be emphasized, though, that this better agreement with the data is rather modest. In this connection it is also worth mentioning that eqs. (33) and (29) representing the direct and total (direct minus exchange) Coulomb energy of a uniformly charged incompressible liquid drop, reproduce our data base of $391^{\star}$ CDE with $a_{\mathrm{C}}$ values of 1321.180 and $1378.886 \mathrm{keV}$ with standard deviations of 386 and $181 \mathrm{keV}$. The introduction of an adjustable additive constant $b$ [ ref. ${ }^{17}$ )], accounting in an approximate way for exchange energy and other effects, lowers the standard deviation with the two parameters $a_{\mathrm{C}}=1425.483 \mathrm{keV}$ and $b=-970.240 \mathrm{keV}$ further to $95 \mathrm{keV}$. With the 17 adjustable coefficients from table 3 eq. (16) reproduces 377 data points ${ }^{\star \star}$ with a standard deviation of $62 \mathrm{keV}$, and the 22 coefficients of eqs. (8) and $(12 \mathrm{a}, \mathrm{b})$ from table 2 reduce this further to $55 \mathrm{keV}$.

Thus, the gross behavior of Coulomb displacement energies is described very well by the liquid drop model, and the various approaches of the shell model are crucial only for the understanding of fine structure details of CDE systematics.

The impressive quality of the two-parameter fit to the data is shown in fig. 1 of ref. $\left.{ }^{16}\right)$.

We conclude with the following remark: in spite of the overall better agreement of eqs. (12a, b) with the data as compared to eq. (16), the latter equation seems to be presently preferable in regions $(7,7)$ and $(8,7)$ to eqs. $(8)$ and $(12 a, b)$ for the prediction of unknown CDE and total ground-state Coulomb energies. This conclusion is based on the much larger statistical errors of the coefficients of eqs. (8) and $(12 \mathrm{a}, \mathrm{b})$ in these regions in table 2 as discussed in sects. 4.2.1 and 4.2.2.

According to ref. ${ }^{5}$ ) (see also end of sect. 4.3.1), the values of $d_{7}$ in both table 2 and table 3 should be considered unrealistically low, and therefore the predictions

* Including ${ }^{3} \mathrm{H}$ and the 7 points with experimental uncertainties exceeding $120 \mathrm{keV}$ (sect. 4.1).

$\star \star$ Excluding 6 points from regions $(3,2),(2,3)$, the ${ }^{3} \mathrm{H}$ point and the 7 points with experimental uncertainties exceeding $120 \mathrm{keV}$ (sect. 4.1). 
of both equations in regions $(7,7)$ and $(8,7)$ appear unreliable. This conclusion is supported by the relatively small value of the ratio $\left[Z_{1}+\frac{1}{2}-\frac{1}{2} \kappa(\beta+\gamma)\right] d /\left(c+\frac{1}{2} \pi\right) \times$ $(1-\kappa \gamma)$ (see sect. 5.1) and the resulting negative value of $\beta_{2}^{2}$, eq. (50), in regions $(7,7)$ and $(8,7)$ as discussed in sect. 5.3.

\subsection{MASS-NUMBER DEPENDENCE OF THE SCALING FACTORS}

We also carried out least-squares adjustments as described in sect. 4.2 for values of the scaling power $\lambda$ increasing by steps of 0.05 in the interval $(0,1)$. The values of $\lambda$ which gave the smallest standard deviation $\sigma$ (called $\lambda_{\text {best }}$ ) and the corresponding $\sigma$-values are given in table 5 for eqs. (8) and $(12 \mathrm{a}, \mathrm{b})$. The situation for eq. (16) is similar.

One observes that the $\lambda_{\text {best }}$ value is near 0 in the $1 \mathrm{p}$ shell, and it increases towards the value 1 expected for saturated nuclear density in heavier nuclei. This increase is not monotonous, and the values of $\lambda_{\text {best }}$ in regions $(4,4)$ and $(6,6)$, particularly the first, are lower than in the preceding regions $(3,3)$ and $(5,5)$. The corresponding fluctuations obtained in combined regions are smaller than in individual regions. The overall $\lambda_{\text {best }}$ for eqs. (8) and $(12 \mathrm{a}, \mathrm{b})$ is 0.85 from region $(3,3)$ onwards, and 0.80 for all the data, with corresponding standard deviations of 46 and $61 \mathrm{keV}$.

The standard deviations obtained for $\lambda_{\text {best }}$ are in most cases smaller by only a few $\mathrm{keV}$ than those obtained for $\lambda=0$ in the $1 \mathrm{p}$ shell and $\lambda=1$ for the $1 \mathrm{~d} 2 \mathrm{~s}$ and the heavier shells. The exception is region $(4,4)$ where $\lambda_{\text {best }}$ is 0.5 rather than 1.0 , and the improvement is from 68 to $44 \mathrm{keV}$ for eqs. (8) and (12a, b) and from 69 to $43 \mathrm{keV}$ for eq. (16).

These findings are in qualitative agreement with the region-dependence of $\lambda_{\text {best }}$ obtained in ref. ${ }^{5}$ ) from the more limited data base available earlier. They are presumably related to deviations of experimental nuclear charge radii from the $A^{1 / 3}$ dependence, as displayed in fig. 2 of ref. ${ }^{18}$ ). However, the study of this connection is beyond the scope of the present work.

Most of the $\sigma_{\text {r.m.s. }}$ values in non-diagonal regions in table 4 are close to those shown in table 2 of ref. ${ }^{5}$ ). The latter were obtained by using different scalings with respect to $N$ and $Z$.

\section{Phenomenological relations to the liquid drop model ${ }^{4}$ )}

In this section we use the similar $A$ - and $Z$-dependence of the shell-model and liquid-drop-model Coulomb-energy expressions to deduce values for the liquid-drop radius and deformation parameters from the shell model coefficients. 
5.1. COMPARISON OF SHELL-MODEL AND LIQUID-DROP MODEL COULOMB-ENERGY EQUATIONS

The Coulomb energy of a spherical nucleus is given in the liquid drop model by

$$
E_{\text {l.d. }}^{\mathrm{C}}(Z, A)=a_{\mathrm{C}} \frac{f(Z)}{A^{1 / 3}}
$$

where

$$
f(Z)=Z^{2}-\kappa Z^{4 / 3}
$$

with

$$
\begin{gathered}
\kappa=5\left(\frac{3}{16 \pi}\right)^{2 / 3}=0.763609 \\
a_{\mathrm{C}}=\frac{3}{5} \frac{e^{2}}{4 \pi \varepsilon_{0}} \frac{1}{r_{0}} .
\end{gathered}
$$

In eq. (32) $e$ is the proton charge, $\varepsilon_{0}$ the permittivity constant of the vacuum, and $r_{0}$ the equivalent uniform radius parameter. The total Coulomb energy of eq. (29) is a difference of direct and exchange Coulomb energies, respectively, given by

$$
E_{\mathrm{ld} .}^{\mathrm{Cdir}}(Z, A)=a_{\mathrm{C}} \frac{Z^{2}}{\mathrm{~A}^{1 / 3}}
$$

and

$$
E_{\text {l.d. }}^{\text {Cexch }}(Z, A)=\kappa a_{\mathrm{C}} \frac{Z^{4 / 3}}{A^{1 / 3}}
$$

Eq. (33) is quadratic in $p$ in all shell regions, with $A^{-1 / 3}$ scaling like eq. (4), but without the pairing term. Eq. (34) is not quadratic in $p$, but the smooth function $Z^{4 / 3}=\left(Z_{1}+p\right)^{4 / 3}$ can be approximated by a quadratic function with deviations in all shell regions less than 0.06 . The corresponding errors in $E_{1 \mathrm{~d}}^{\mathrm{Cexch}}$ are a few keV. With $Z=Z_{1}+p$ one obtains

$$
\begin{aligned}
E_{1 . d .}^{\mathrm{C}}\left(Z_{1}+p, A\right) & \left(\frac{A_{1}}{A}\right)^{1 / 3} \frac{a_{\mathrm{C}}}{A_{1}^{1 / 3}}\left[\left(Z_{1}^{2}-\kappa \alpha\right)+\left(\left(2 Z_{1}+1\right)-\kappa(\beta+\gamma)\right) p+(1-\kappa \gamma) p(p-1)\right], \\
& \Delta E_{\text {l.d. }}^{\mathrm{C}}\left(Z_{1}+p, A\right) \approx\left(\frac{A_{1}}{A}\right)^{1 / 3} \frac{a_{\mathrm{C}}}{A_{1}^{1 / 3}}\left[\left(2 Z_{1}+1-\kappa(\beta+\gamma)\right)+2(1-\kappa \gamma) p\right] .
\end{aligned}
$$

Here, the coefficients $\alpha, \beta$ and $\gamma$ are defined by the expansion of $Z^{4 / 3}$ in orthogonal polynomials in $p$ which we rewrite as

$$
\left(Z_{1}+p\right)^{4 / 3} \approx \alpha+\beta p+\gamma p^{2} .
$$

The explicit expressions and a table of numerical values of $\alpha, \beta$ and $\gamma$ are given in appendix A.4. 
Eqs. (35) and (36) have the form of the shell model eqs. (4) and (8) without the $\delta_{\text {odd }}$ and $\delta_{\text {even }}$ pairing terms, with corresponding shell model coefficients given by

$$
\begin{aligned}
E_{0} & =a_{\mathrm{C}} \frac{Z_{1}^{2}-\kappa \alpha}{A_{1}^{1 / 3}}, \\
c+\frac{1}{2} \pi & =a_{\mathrm{C}} \frac{2 Z_{1}+1-\kappa(\beta+\gamma)}{A_{1}^{1 / 3}}, \\
d & =2 a_{\mathrm{C}} \frac{1-\kappa \gamma}{A_{1}^{1 / 3}} .
\end{aligned}
$$

Eq. (36) predicts the main features of the systematics observed in figs. 1 and 2, excluding fine structure effects like odd-even variations and magic-number discontinuities due to shell structure. We discussed its quantitative fit to the data in sect. 4.4.

Eqs. (39) and (40) predict the following relation between the shell model coefficients:

$$
\frac{c+\frac{1}{2} \pi}{d}=\frac{Z_{1}+\frac{1}{2}-\frac{1}{2} \kappa(\beta+\gamma)}{1-\kappa \gamma} .
$$

The coefficients of tables 2 and 3 , however, satisfy the inequality $\left(c+\frac{1}{2} \pi\right)(1-\kappa \gamma)<$ $\left[Z_{1}+\frac{1}{2}-\frac{1}{2} \kappa(\beta+\gamma)\right] d$ except for region $(7,7)$. Therefore, eq. (4) (for even- $Z$ nuclei) cannot be identified with eq. (29) throughout a shell region.

On the other hand, since the experimental Coulomb displacement cncrgics determine only the coefficients $c, \pi$ and $d$ but not $E_{0}$, one still has the freedom to fix $E_{0}$ in such a way that eq. (4) coincides with eq. (29) on the $Z$-boundaries of the shell region. As a matter of fact, the requirement $E_{\text {shell mod }}^{\mathrm{C}}(Z, A)=E_{\text {l.d. } \bmod }^{\mathrm{C}}(Z, A)$ for both $Z=Z_{1}$ and $Z=Z_{2}=Z_{1}+\delta$ results in a pair of equations allowing the determination of $a_{c}$ and $E_{0}$ for given values of $c+\frac{1}{2} \pi$ and $d$.

Equating eqs. (29) and (4) for $p=0$ and for $p=\delta$, one obtains the following two equations:

$$
\begin{aligned}
& \frac{f\left(Z_{1}\right)}{A_{1}^{1 / 3}} a_{\mathrm{C}}-E_{0}=0, \\
& \frac{f\left(Z_{2}\right)}{A_{1}^{1 / 3}} a_{\mathrm{C}}-E_{0}=\delta\left(c+\frac{1}{2} \pi\right)+\frac{1}{2} \delta(\delta-1) d,
\end{aligned}
$$

having the solutions

$$
\begin{aligned}
& a_{C}=\left[\delta\left(c+\frac{1}{2} \pi\right)+{ }_{2}^{1} \delta(\delta-1) d\right] \frac{A_{1}^{1 / 3}}{f\left(Z_{2}\right)-f\left(Z_{1}\right)}, \\
& E_{0}-\left[\delta\left(c+\frac{1}{2} \pi\right)+\frac{1}{2} \delta(\delta-1) d\right] \frac{f\left(Z_{1}\right)}{f\left(Z_{2}\right)-f\left(Z_{1}\right)} .
\end{aligned}
$$

The analogous equations for the $1 \mathrm{p}$ shell with the constant radius $R=r_{0}(16)^{1 / 3}$ are obtained by substituting the values $Z_{1}=2, A_{1}=16$ and $\delta=6$ into eqs. (42)-(45). 
Numerical values for the quantities $a_{C}$ and $E_{0}$ calculated from the r.h.s. of eqs. (44) and (45), with the coefficients of table 2 for the various shell regions, are given in tables 6 and 8 . Their physical implications are discussed in the following two sections.

\subsection{SHELL-MODEL COULOMB DISPLACEMENT ENERGIES AND THE LIQUID-DROP NUCLEAR CHARGE RADIUS PARAMETER $r_{0}$}

From eqs. (44) and (32) one can extract an expression for the parameter $r_{0}$,

$$
r_{0}=\frac{1}{\delta\left(c+\frac{1}{2} \pi\right)+\frac{1}{2} \delta(\delta-1) d} \frac{f\left(Z_{2}\right)-f\left(Z_{1}\right)}{A_{1}^{1 / 3}} \frac{3}{5} \frac{e^{2}}{4 \pi \varepsilon_{0}} .
$$

Using $\frac{3}{5}\left(e^{2} / 4 \pi \varepsilon_{0}\right)=0.863974 \mathrm{MeV} \cdot \mathrm{fm}\left[\right.$ ref. $\left.\left.^{19}\right)\right]$ and the values of the energy parameters from table 2 we calculated the values of $r_{0}$ for all shell regions.

The results are given in the third column of table 6 . Experimental values ${ }^{13,14}$ ) of the parameter $r_{0}$ of nuclei in the corresponding shell regions are shown for comparison in the fifth column of the table. In most shell regions the agreement is better than 0.05 fermi. The discrepancy of $0.13 \mathrm{fm}$ occurs in region $(2,2)$, presumably indicating the inadequacy of the liquid drop model for such light nuclei.

Neglecting the exchange energy (34) by setting $\kappa=0$ in eq. (30) reduces eq. (46) to

$$
r_{0}=\frac{1}{c+\frac{1}{2} \pi+\frac{1}{2}(\delta-1) d} \frac{2 Z_{1}+\delta}{A_{1}^{1 / 3}} \frac{3}{5} \frac{e^{2}}{4 \pi \varepsilon_{0}} .
$$

The calculated values of the r.h.s, of eq. (47) are given in the fourth column of table 6. They are higher than the corresponding experimental values by about $0.1 \mathrm{fm}$, thus confirming the essential role of the exchange energy in obtaining realistic values for $r_{0}\left[\right.$ ref. $^{20}$ )] when using the liquid drop model.

TABLE 6

Nuclear charge radius parameters

\begin{tabular}{ccccc}
\hline Region & $a_{\mathrm{C}} \begin{array}{c}{[\text { eq. (44)] }} \\
(\mathrm{keV})\end{array}$ & $\begin{array}{c}r_{\mathrm{o}}\left[\begin{array}{c}\text { eq. (46)] } \\
(\mathrm{fm})\end{array}\right. \\
r_{0}\left[\begin{array}{c}\text { eq. (47)] } \\
(\mathrm{fm})\end{array}\right.\end{array}$ & $\begin{array}{c}\left.r_{0} \text { [exp. }^{\mathrm{a}}\right) \\
(\mathrm{fm})\end{array}$ \\
\hline$(2,2)$ & 682.439 & 1.27 & 1.53 & 1.40 \\
$(4,3)+(4,3)$ & 666.139 & 1.30 & 1.42 & 1.33 \\
$(5,5)+(6,5)$ & 690.276 & 1.27 & 1.35 & 1.26 \\
$(6,6)+(7,6)$ & 695.774 & 1.25 & 1.31 & 1.24 \\
$(7,7)+(8,7)$ & 674.679 & 1.28 & 1.28 & 1.20 \\
\hline
\end{tabular}

a) These values are averages of the experimental $r_{0}$ values of all nuclei in the region, calculated from the r.m.s. charge radii given in ref. ${ }^{14}$ ). 
5.3. SHELL-MODEL COULOMB DISPLACEMENT ENERGIES AND THE LIQUID-DROP NUCLEAR CHARGE DEFORMATION PARAMETER $\beta_{2}$

Using eqs. (44) and (45) one can write the Coulomb energy, eq. (4), of an even- $Z$ nucleus as

$$
\begin{aligned}
& E_{\mathrm{s} . \mathrm{m} .}^{\mathrm{C}}\left(Z_{1}+p, A\right) \\
& \quad=\frac{a_{\mathrm{C}} f(Z)}{A^{1 / 3}}\left[\begin{array}{c}
{\left[f\left(Z_{2}\right)-f\left(Z_{1}\right)\right]\left[\left(c+\frac{1}{2} \pi\right) p+\frac{1}{2} d p(p-1)\right]} \\
1+\frac{-\left[f(Z)-f\left(Z_{1}\right)\right]\left[\left(c+\frac{1}{2} \pi\right) \delta+\frac{1}{2} d \delta(\delta-1)\right]}{f(Z)\left[\left(c+\frac{1}{2} \pi\right) \delta+\frac{1}{2} d \delta(\delta-1)\right]}
\end{array}\right] .
\end{aligned}
$$

The factor multiplying the large square brackets is the liquid-drop Coulomb-energy expression (29). The second term inside the brackets vanishes for $p=0$ and $p=\delta$, as it should according to the requirement made above that the shell model and liquid-drop model values of the Coulomb energy coincide for closed proton shells.

Neglecting the deformation dependence of the exchange energy, the Coulomb energy of a deformed nucleus with a small deformation is given to second order in the deformation parameter $\beta_{2}$ by ${ }^{21}$ )

$$
E_{\text {l.d. }}^{\mathrm{C}}\left(Z, A, \beta_{2}\right)=a_{\mathrm{C}} \frac{Z^{2}}{A^{1 / 3}}\left[1-\frac{\beta_{2}^{2}}{4 \pi}\right]-\kappa a_{\mathrm{C}} \frac{Z^{4 / 3}}{A^{1 / 3}} .
$$

Equating eqs. (48) and (49) one obtains an expression for the liquid-drop nuclear charge deformation parameter in a given shell region in terms of the shell model parameters as

$$
\begin{aligned}
& \beta_{2}^{2}\left(Z_{1}+p, A\right) \\
& =-4 \pi \frac{\left[f\left(Z_{2}\right)-f\left(Z_{1}\right)\right]\left[\left(c+\frac{1}{2} \pi\right) p+\frac{1}{2} d p(p-1)\right]}{-\left[f(Z)-f\left(Z_{1}\right)\right]\left[\left(c+\frac{1}{2} \pi\right) \delta+\frac{1}{2} d \delta(\delta-1)\right]} \\
& Z^{2}\left[\left(c+\frac{1}{2} \pi\right) \delta+\frac{1}{2} d \delta(\delta-1)\right]
\end{aligned}
$$

The r.h.s of eq. (50) attains its maximum value within a shell region at a value $p=p_{\max }$ somewhat lower than the middle of the major proton shell $\frac{1}{2} \delta$ [see also eq. (A.22), appendix A.4]. The maximum value of the deformation in the region, $\beta_{2 \max }$, is obtained from eq. (50) by substituting respectively $p_{\max }$ and $Z_{\max }=Z_{1}+p_{\max }$ for $p$ and $Z$.

The values of $p_{\max }$ (nearest integer) and the values of $\beta_{2 \max }$ calculated from eq. (50) with the coefficients of table 2 are given in table 7 . Experimental values of $p_{\max }$ and $\beta_{2 \max }$ [ref. $\left.\left.{ }^{22}\right)\right]$ for the corresponding regions are also shown for comparison.

One observes that the calculated values of $Z_{\max }$ are quite close to the experimental values, but the calculated values of $\beta_{\max }$ are smaller than the experimental ones, particularly for the highly deformed nuclei in regions $(2,2),(5,5),(6,5),(6,6)$ and $(7,6)$. 
TABLE 7

Nuclear charge deformation parameters

\begin{tabular}{ccccc}
\hline Region & $\begin{array}{c}Z(\max ) \\
\text { eq. (50) }\end{array}$ & $\begin{array}{c}Z(\max ) \\
\left.\text { exp. }{ }^{22}\right)\end{array}$ & $\begin{array}{c}\beta_{2 \max } \\
\text { eq. }(50)\end{array}$ & $\begin{array}{c}\beta_{2 \max } \\
\left.\text { exp. }{ }^{22}\right)\end{array}$ \\
\hline$(2,2)$ & 3 & 4 & 0.26 & $1.13\left({ }^{10} \mathrm{Be}\right)$ \\
$(3,3)+(4,3)$ & 11 & 10 & 0.43 & $0.73\left({ }^{20} \mathrm{Ne}\right)$ \\
$(4,4)+(5,4)$ & 23 & 24 & 0.17 & $0.34\left({ }^{48} \mathrm{Cr}\right)$ \\
$(5,5)+(6,5)$ & 36 & 38 & 0.13 & $0.43\left({ }^{78} \mathrm{Sr}\right), 0.42\left({ }^{102} \mathrm{Zr}\right)$ \\
$(6,6)+(7,6)$ & 59 & 64 & 0.03 & $0.29\left({ }^{128} \mathrm{Ce}\right), 0.35\left({ }^{160} \mathrm{Gd}\right)$ \\
$(7,7)+(8,7)$ & 99 & 98 & $\left.{ }^{a}\right)$ & $0.30\left({ }^{252} \mathrm{Cf}\right)$ \\
\hline
\end{tabular}

a) $\beta_{2 \max }^{2}=-0.074$

The reduction of CDE due to deformation in highly deformed rare earth nuclei ${ }^{23}$ ) is of the order of $100 \mathrm{keV}$. It can be described in an average way by eqs. (8) and $(12 \mathrm{a}, \mathrm{b})$ of the spherical shell model, with a slight adjustment of the coefficients. In fact, the data of highly deformed nuclei in region $(7,6)$ fit nicely with the rest of the systematics in fig. 2 [the same applies to fig. 1 of ref. ${ }^{16}$ )], and the $\sigma_{\text {r. m.s. value }}$ for this region in table 4 has similar magnitude to the other regions. On the other hand specific small deformation effects cannot be described in this way. Deformation effects are explicitly considered in ref. ${ }^{24}$ ).

The r.h.s. of eq. $(50)$ for regions $(7,7)$ and $(8,7)$ is negative. This invalidates the above correspondence between the shell model and the liquid drop model in these regions. As mentioned in sect. 4.4 this behavior is presumably due to the unreliably low value of $d_{7}$ in tables 2 and 3. Indeed, according to eqs. (A.21) and (A.22), in order to have a positive $\beta_{\max }^{2}$ value with the $c_{7}+\frac{1}{2} \pi_{7}$ value from table 2 , the value of $d_{7}$ should exceed $253 \mathrm{keV}$ for local scaling (550 keV for global scaling). It is perhaps not unreasonable to assume that the low value of $d_{7}$ is due to the fact that it was determined essentially from deformed nuclei [the isotopes of $T h$ and $U$ in region $(8,7)]$.

\section{Total ground-state Coulomb energies}

Total ground-state Coulomb energies can be calculated from eq. (4) with the numerical values of the coefficients $E_{0}, c, \pi$ and $d$ from tables 2 and 8 . The last three coefficients were determined directly from experimental Coulomb displacement energies, whereas $E_{0}$ was calculated above from the other coefficients using the requirement that the Coulomb energy of closed-proton-shell nuclei behaves like that of a charged liquid drop.

Closed-proton-shell nuclei are situated on the boundary of two shell regions in the $(N, Z)$ plane. Their Coulomb energy can be calculated from the coefficients of both shell regions, as either $\left(A_{1} / A\right)^{\lambda / 3} E_{0}$ with the coefficient $E_{0}$ of the higher shell region, or $\left(A_{1} / A\right)^{\lambda / 3}\left[E_{0}+\delta\left(c+\frac{1}{2} \pi\right)+\frac{1}{2} \delta(\delta-1) d\right]$ with the coefficients $E_{0}, c, \pi$ and 
TABLE 8

Values of $E_{0}$ (in $\mathrm{keV}$ ) calculated from the coefficients in table 2

\begin{tabular}{|c|c|c|c|}
\hline Region & $\begin{array}{c}E_{0} \text { from the } \\
\text { higher region }\end{array}$ & $\begin{array}{l}E_{0} \text { from the } \\
\text { lower region }\end{array}$ & $\begin{array}{c}E_{0} \text { from } \\
\Delta E^{\mathrm{C}\left({ }^{3} \mathrm{He}\right)}\end{array}$ \\
\hline \multicolumn{4}{|c|}{ (a) Regional scaling ${ }^{\text {a) }}$} \\
\hline$(2,2)$ & 562.188 & - & 903.133 \\
\hline$(3,3)$ & 13689.030 & 14023.995 & 14364.940 \\
\hline$(4,4)$ & 71295.287 & 69837.487 & 70335.501 \\
\hline$(5,5)$ & 129738.110 & 127814.993 & 126957.037 \\
\hline$(6,6)$ & 353665.399 & 350870.805 & 348578.485 \\
\hline$(7,7)$ & 795258.087 & 820123.275 & 815809.676 \\
\hline$(8,8)$ & - & 1644265.572 & 1662075.405 \\
\hline \multicolumn{4}{|c|}{ (b) Global scaling ${ }^{a}$ ) } \\
\hline$(2,2)$ & 562.188 & - & 903.133 \\
\hline$(3,3)$ & 13689.030 & 14023.995 & 14364.940 \\
\hline$(4,4)$ & 96762.591 & 94784.052 & 95459.961 \\
\hline$(5,5)$ & 196980.657 & 194060.799 & 192758.169 \\
\hline$(6,6)$ & 651457.235 & 646309.548 & 642087.059 \\
\hline$(7,7)$ & 1727492.016 & 1781505.191 & 1772135.015 \\
\hline$(8,8)$ & - & 4121596.594 & 4166239.594 \\
\hline
\end{tabular}

a) The scaling parameters for each region are the same as in table 2 .

$d$ of the lower shell region. The two values are given in the second and third column of table 8 .

The $E_{0}$ coefficients can also be calculated $\left.{ }^{5}\right)$ by starting from $E_{0}(2,2)=E^{\mathrm{C}}\left({ }^{4} \mathrm{He}\right)$ and successively adding to it the values of $\left(A_{1} / A\right)^{\lambda / 3}\left[\delta\left(c+\frac{1}{2} \pi\right)+\frac{1}{2} \delta(\delta-1) d\right]$ of the corresponding shell regions in table 2. The starting value $E^{\mathrm{C}}\left({ }^{4} \mathrm{He}\right)=\Delta E^{\mathrm{C}}\left({ }^{4} \mathrm{He}\right)$ is ohtained hy multiplying the experimental value $\Delta F^{\mathrm{C}}\left({ }^{3} \mathrm{He}\right)=763.749 \pm 0.042 \mathrm{keV}$ [ref. ${ }^{15}$ )] by $1.1825 \pm 0.0189$ which is the ratio of the experimental values of charge radii of ${ }^{3} \mathrm{He}$ and ${ }^{4} \mathrm{He}$ [ref. $\left.\left.{ }^{14}\right)\right]$. The resulting $E_{0}$ values are given in the last column of table 8 .

There are considerable differences between corresponding values of $E_{0}$ obtained by the three procedures. According to eqs. (29) and (32) the first two values are inversely proportional to the two different values of the nuclear charge radius parameter $r_{0}$ in the two regions as given in table 6 . The relative differences of both the $E_{0}$ and the $r_{0}$ values are the same. The third value was obtained by a procedure which is least dependent on analogies to a liquid drop. However, its accumulated error due to the statistical errors and correlations of the coefficients of table 2 might be larger.

\section{Conclusions}

The present work updates and extends a previous phenomenological study ${ }^{5}$ ) of Coulomb displacement energies based on the shell model. Shell-model equations 
(sect. 2) are applied to a detailed interpretation of CDE systematics (sect. 3) and are adjusted (sect. 4.2) to CDE of ground states from two recent comprehensive compilations ${ }^{15,16}$ ) and more recent literature. These latter values, supplementing the compilations, are summarized in table A.2.

A major motivation was to use the adjusted coefficients as input in the construction of nuclear mass equations. A second motivation was to use the results for predictions of unknown CDE, excitation energies of IAS, and IMME coefficients, particularly for nuclei far from stability. In order to increase the predictive power of the equations we adjusted them to the widest data base available to us (see sect. 4.1), and we checked the physical plausibility of the adjusted values using as a guideline explicit expressions of shell-model Coulomb-interaction matrix elements (sect. 4.3.1). Values contradicting the latter, like negative Coulomb pairing energies, were rejected in the final analysis (tables 2 and 3 ).

With 22 adjustablc coefficients for 12 shell regions (table 1) the equations reproduce $377 \mathrm{CDE}$ with a standard deviation of $55 \mathrm{keV}$. A detailed study of the residuals (sect. 4.3.2 and table 4) shows that they are not evenly distributed. In the first place, they are as a rule larger for light nuclei, where the interparticle distances are smaller and nuclear structure effects are expected to be more pronounced. Residuals of nuclear mass equations behave in a similar way.

Secondly, the residuals are conspicuously large and positive (calculated CDE are too high) for exotic light proton-rich nuclei in the vicinity of the proton drip-line. This reflects similar deviations from the CDE systematics of fig. 1 for these nuclei (sect. 3), which are presumably due to Thomas-Ehrman shifts. Thus, predictions of CDE of such nuclei using the present results should be considered less reliable than in other regions. It would be interesting to find out if regular deviations due to particle instabilities occur as well near the neutron drip line, where there are presently no experimental data available.

Another region where the present results should be considered unreliable is for $Z \geqslant 82$. This is indicated by the rather low value of $d$, in tables 2 and 3 (sects. 4.3.1, $4.4,5.1$ and particularly 5.3), which is presumably due to the fact that it was determined essentially from the very few data in region $(8,7)$ belonging to deformed nuclei.

On the other hand, the deviations in regions of deformation are statistically similar to those for non-deformed nuclei. This is to be expected, considering the small magnitude of deformation effects on $\mathrm{CDE}^{23}$ ) (sect. 5.3).

Coulomb displacement energies can be calculated from the adjusted coefficients of tables 2 and 3. On the other hand, to calculate total ground state Coulomb energies one needs the value of the Coulomb energy of the closed shells, $E_{0}$, as well. In ref. ${ }^{5}$ ) the values of $E_{0}$ for all shell regions were calculated by starting from a renormalized experimental value for ${ }^{4} \mathrm{He}$, and adding to it successively the interaction of valence protons in the higher closed major shells. The latter were calculated from the adjusted values of the $c, \pi$ and $d$ coefficients. In the present 
work we exploited the similar $p$-dependence of the liquid-drop and shell-model Coulomb-energy expressions to calculate $E_{0}$ in each region in terms of its $c, \pi$ and $d$ coefficients, by imposing the requirement that the two expressions coincide on the $Z$-boundarics of the region (sect. 5.1). As a matter of fact, this can be done in two different ways (sect. 6). The three values of $E_{0}$ thus obtained (table 8) are not equal, which should serve as a warning against the indiscriminate application of the results to the construction of nuclear mass equations.

The above summarizes the main practical conclusions from the present work. In addition, a very regular isodiapheric systematics of CDE is presented (sect. 3). This is simpler to use as a predictive tool than the equations, and it is presumably as reliable for interpolations and short-distance extrapolations.

Furthermore, we considered briefly the approximations used in deriving the equations (sect. 2 and appendix A.2), and gained a better understanding of their relations to other shell model approaches (sect. 2, 4.2.2, 4.4 and appendix A.1). We also pursued the above-mentioned formal analogy between the shell-model and liquid-drop-model Coulomb-energy equations to derive phenomenological "shell model" values, calculated from the adjusted shell-model coefficients, for the liquiddrop charge radius and deformation parameters (sect. 5 and appendix A.4). The resulting radii for regions $(3,3)$ to $(7,6)$ agree with the experimental radii to better than $0.05 \mathrm{fm}$ (table 6 ), but the resulting deformation parameters are too small (table 7).

Stimulating discussions with K.T. Hecht are highly appreciated. We also thank the referee for useful suggestions. One of the authors (N.Z.) thanks the Department of Physics, The University of Michigan, for its warm hospitality and for financial support. This work was supported in part by the U.S. National Science Foundation under grants PHY8605907 and PHY8911831 and by the Visitor Program, Department of Physics, The University of Michigan.

\section{Appendix}

\section{A.1. DIFFERENCES BETWEEN THE CARLSON-TALMI ${ }^{3}$ ) AND THE HECHT ${ }^{\text {") }}$ ) GROUND-STATE COULOMB ENERGY EQUATIONS}

The fourth and fifth columns of table A.1 show the maximum values (in units of $\pi$ ) of the difference $\Delta^{\mathrm{CT}-\mathrm{H}}=E_{\mathrm{CT}}^{\mathrm{C}}-E_{\mathrm{H}}^{\mathrm{C}}$ for states of lowest isospin $T$ and lowest seniority $v$ compatible with this value of $T$ for mixed $j^{a}$ configurations. The second and third columns of table A.1 show the $a, T, v$ and $t$ values for the maximum difference. The difference is the same for any two particle-hole-conjugate nuclei. The $a$-values given are for $T_{z} \geqslant 0$. The $t$ denotes the reduced isospin quantum number, and $\delta=2 j+1$. 
TABLE A.1

Differences between the Carlson-Talmi and the Hecht g.s. Coulomb energy equations

\begin{tabular}{|c|c|c|c|c|}
\hline \multirow[b]{2}{*}{ Parity type } & \multicolumn{2}{|c|}{$(a, T, v, t)$} & \multicolumn{2}{|c|}{$\Delta^{\mathrm{CT}-\mathrm{H}}(\pi)$} \\
\hline & $\begin{array}{c}\frac{1}{2} \delta \text { even } \\
\left(j=\frac{3}{2}, \frac{7}{2}, \frac{11}{2}, \ldots\right)\end{array}$ & $\begin{array}{c}\frac{1}{2} \delta \text { odd } \\
\left(j=\frac{1}{2}, \frac{5}{2}, \frac{9}{2}, \ldots\right)\end{array}$ & $\begin{array}{c}\frac{1}{2} \delta \text { even } \\
\left(j=\frac{3}{2}, \frac{7}{2}, \frac{11}{2}, \ldots\right)\end{array}$ & $\begin{array}{c}\frac{1}{2} \delta \text { odd } \\
\left(j=\frac{1}{2}, \frac{5}{2}, \frac{9}{2}, \ldots\right)\end{array}$ \\
\hline \multirow{2}{*}{ even-even } & \multirow{2}{*}{$(\delta, 0,0,0)$} & \multirow{2}{*}{$(\delta \pm 2,0,0,0)$} & $\delta^{2}$ & $(\delta+2)(\delta-2)$ \\
\hline & & & $24(\delta+1)$ & $24(\delta+1)$ \\
\hline \multirow{2}{*}{ odd- $a$} & \multirow{2}{*}{$\left(\delta+1, \frac{1}{2}, 1, \frac{1}{2}\right)$} & \multirow{2}{*}{$\left(\delta-1, \frac{1}{2}, 1, \frac{1}{2}\right)$} & $\delta^{2}$ & $(\delta+2)(\delta-2)$ \\
\hline & & & $24(\delta+1)$ & $24(\delta+1)$ \\
\hline \multirow{2}{*}{ odd-odd } & \multirow{2}{*}{$(\delta \pm 2,0,2,0)$} & \multirow{2}{*}{$(\delta, 0,2,0)$} & $\delta(\delta-4)$ & $(\delta-2)^{2}$ \\
\hline & & & $24(\delta+1)$ & $24(\delta+1)$ \\
\hline \multirow{2}{*}{ odd-odd } & \multirow{2}{*}{$(\delta, 1,2,1)$} & \multirow{2}{*}{$(\delta+2,1,2,1)$} & $\delta-4$ & $(\delta-1)(\delta-2)$ \\
\hline & & & 20 & $20(\delta+1)$ \\
\hline
\end{tabular}

\section{A.2. THE ISOSCALAR, ISOVECTOR AND ISOTENSOR COULOMB ENERGIES}

The values of the Coulomb encrgy of the $T_{z}= \pm T$ ground-state members of an isospin multiplet do not allow a unique determination of the isoscalar and isotensor Coulomb energies separately. In fact one has

$$
\begin{aligned}
E^{\mathrm{C}(1)} & =\frac{E^{\mathrm{C}}(T,-T)-E^{\mathrm{C}}(T, T)}{2 T}, \\
E^{\mathrm{C}(0)}+T(2 T-1) E^{\mathrm{C}(2)} & =\frac{1}{2}\left[E^{\mathrm{C}}(T,-T)+E^{\mathrm{C}}(T, T)\right],
\end{aligned}
$$

where $E^{\mathrm{C}}(T, \pm T)$ denote the Coulomb energies $E^{\mathrm{C}}\left(T, T_{z}= \pm T\right)$ of the ground-state members of the multiplet. This explains why it is possible to have different pairing terms for $E^{\mathrm{C}(0)}$ and $E^{\mathrm{C}(2)}$ in diagonal regions in ref. ${ }^{5}$ ) and in the present work, both of which are based on the Carlson-Talmi eq. (4) for ground-state energies.

A.2.1. Diagonal regions. Eqs. (5)-(7) were derived as follows: the Carlson-Talmi eq. (4) can be written in a diagonal region as

$$
\begin{aligned}
\left(\frac{A}{A_{1}}\right)^{\lambda / 3} E^{\mathrm{C}}\left(A_{1}+a, T, T_{z}\right)= & {\left[E_{0}+\frac{1}{2} a\left(c+\frac{1}{2} \pi\right)+\left(\frac{1}{8} a(a-2)+\frac{1}{6} T(T+1)\right) d\right] } \\
& -T_{z}\left[c+\frac{1}{2} \pi+\frac{1}{2}(a-1) d\right]+\left(3 T_{z}^{2}-T(T+1)\right)\left[\frac{1}{6} d\right] \\
& -\delta_{\text {odd }\left(\frac{1}{2} a-T_{z} \frac{1}{2} \pi .\right.}
\end{aligned}
$$

The first two lines have the form of an IMME, eq. (1), indicating a possible "natural" choice of $E^{\mathrm{C}(0)}, E^{\mathrm{C}(1)}$ and $E^{\mathrm{C}(2)}$ as the respective square brackets on the r.h.s. of eq. (A.3). The last term, however, is not a quadratic function of $T_{z}$. 
However, one can write quadratic functions of $T$, which coincide with $\delta_{\text {ndd }\left(\frac{1}{2} a-T_{z}\right)}$ for $T_{z}= \pm T$ [see also ref. ${ }^{25}$ )]. One such choice is

$$
\begin{aligned}
& \delta_{\text {odd }\left(\frac{1}{2} a-T z\right)}\left(\text { for } T_{z}= \pm T\right) \\
&=\frac{1}{2} \delta_{\text {odd } a}\left(1-(-1)^{\frac{1}{2} a-T}\left(T_{z} / T\right)\right)+\frac{1}{2} \delta_{\text {even } a}\left(1-(-1)^{\frac{1}{2} a-T}\left(T_{z}^{2} / T^{2}\right)\right) \\
&=\left[\frac{1}{2}-\delta_{\text {even } a}(-1)^{\frac{1}{2} a-T}(T+1) / 6 T\right] \\
&-T_{z}\left[\delta_{\text {odd } a}(-1)^{\frac{1}{2} a-T} / 2 T\right]+\left(3 T_{z}^{2}-T(T+1)\right)\left[-\delta_{\text {even } a}(-1)^{\frac{1}{2} a-T} / 6 T^{2}\right] .
\end{aligned}
$$

Substituing eq. (A.4) into (A.3) one obtains an expression which has the form of an IMME. The corresponding coefficients of this expression are given by eqs. (5)-(7), which we adopted in the present work.

A.2.2. Non-diagonal regions ${ }^{12}$ ). In non-diagonal regions like those considered in sect. 2.3 one has in the notation used there

$$
\begin{aligned}
\left(\frac{A}{A_{1}}\right)^{\lambda / 3} E^{\mathrm{C}}(T, T)= & E_{0}+p_{0}\left(c_{1}+\frac{1}{2} \pi_{1}\right)+\frac{1}{2} p_{0}\left(p_{0}-1\right) d_{1}-\delta_{p_{0} \text { odd } \frac{1}{2} \pi_{1}} \\
\left(\frac{A}{A_{1}}\right)^{\lambda / 3} E^{\mathrm{C}}(T,-T)= & E_{0}+\delta\left(c_{1}+\frac{1}{2} \pi_{1}\right)+\frac{1}{2} \delta(\delta-1) d_{1} \\
& +n_{0}\left(c_{2}+\frac{1}{2} \pi_{2}\right)+\frac{1}{2} n_{0}\left(n_{0}-1\right) d_{2}-\delta_{n_{0} \text { odd } 2} \pi_{2} .
\end{aligned}
$$

The interaction $E^{C(1)}$, eq. (10), is obtained from eq. (A.1) using eqs. (A.5a) and (A.5b).

The isoscalar Coulomb interaction $E^{\mathrm{C}(0)}$ of a $T$-multiplet is given by the expectation value of the isoscalar Coulomb hamiltonian, $\sum_{i j}\left(\frac{1}{4}+\frac{1}{3}\left(\boldsymbol{t}_{i} \cdot \boldsymbol{t}_{j}\right)\right) e^{2} / r_{i j}$ where the summation extends over all nucleon pairs, in any of the $(2 T+1)$ members of the multiplet. We shall calculate its expectation value in the $T_{z}=T$ ground-state member of the multiplet.

In a non-diagonal region the calculation can be carried out in a $n p$ scheme, using the value $\left(\boldsymbol{t}_{i} \cdot \boldsymbol{t}_{j}\right)=\frac{1}{4}$ for identical nucleons and $\left(\boldsymbol{t}_{i} \cdot \boldsymbol{t}_{j}\right)=-\frac{1}{4}$ for a neutron-proton pair. Furthermore, in the approximation of equal values for corresponding parameters in neighboring subshells (sect. 2.1), one can proceed as if each major shell was comprised of one subshell. For the configuration $\nu j_{1}^{2 j_{1}+1} j_{2}^{n_{0}} \pi j_{1}^{p_{0}}$, with each subshell in a state of lowest seniority, one obtains by summing all intrashell and intershell interactions of the neutron and proton groups the expression

$$
\begin{aligned}
E^{\mathrm{C}(0)}= & E_{0}+\frac{1}{2}\left(\delta+p_{0}\right) c_{1}+\frac{1}{2} n_{0} c_{2}+\frac{1}{6}\left(\delta+p_{0}-\delta_{p_{0} \text { odd }}\right) \pi_{1}+\frac{1}{6}\left(n_{0}-\delta_{n_{0} \text { odd }}\right) \pi_{2} \\
& +\frac{1}{6}\left[\delta(\delta-1)+p_{0}\left(p_{0}-1\right)\right] d_{1}+\frac{1}{6} p_{0} \delta I_{11}^{0}+\frac{1}{6} n_{0}\left(n_{0}-1\right) d_{2} \\
& +\frac{1}{6} n_{0}\left(p_{0}-\delta\right) I_{12}^{0}+\frac{1}{6} \delta_{n_{0} \text { odd }} \delta_{p_{0} \text { odd }} I_{12}^{\prime},
\end{aligned}
$$

where $\frac{1}{6} I_{11}^{0}$ is the antisymmetrized (direct minus exchange) monopole interaction between a neutron and a proton in subshell $j_{1}$, and $\frac{1}{6} I_{12}^{\prime}$ is the higher- $k(k \geqslant 1)$ multipole interaction between the $j_{1}^{p_{0}}$ protons and $j_{2}^{n_{0}}$ neutrons in an odd-odd nucleus. 
Using the relation

$$
I_{1,1}^{0}=\frac{2 j_{1}}{2 j_{1}+1} d_{1}+\frac{1}{2 j_{1}+1} \pi_{1}
$$

obtained from eqs. (18)-(20) one observes that the r.h.s. of eq. (A.6) is equal to eq. (9) plus the additional higher-multipole term with $I_{12}^{\prime}$.

Having calculated $E^{\mathrm{C}(0)}$, one obtains the isotensor Coulomb energy $E^{\mathrm{C}(2)}$ by using eqs. (A.2) and (A.5a, b). The resulting expression is equal to eq. (11) plus an additional higher-multipole term $\left[-\delta_{\text {odd } n_{0}} \delta_{\text {odd } p_{0}} \frac{1}{3} I_{12}^{\prime}\right] / 2 T(2 T-1)$.

Finally, the expression of $\Delta E^{\mathrm{C}}$, calculated from $E^{\mathrm{C}(1)}$ and $E^{\mathrm{C}(2)}$ according to eq. (2) is equal to eq. (12a) plus an additional $\left(\delta_{\text {odd } n} \delta_{\text {odd } p} I_{12}^{\prime}\right) / 2 T$ term for $n$-rich nuclei, and to eq. (12b) plus an additional $\left(-\delta_{\text {even } n} \delta_{\text {even } p} I_{12}^{\prime}\right) I / 2 T$ term for $p$-rich ones.

We now consider these $I_{12}^{\prime}$ terms. Contrary to the other coefficients $E_{0}, c, \pi, d$ and $I_{12}^{0}$ which depend only on the $n l j$ single-proton quantum numbers, the $I_{12}^{\prime}$ coefficient depends in addition on the total angular momentum $J$, and also depends weakly on the numbers of nucleons in the subshells. For odd subshells in states of seniority $v=1$ it can be written as ${ }^{8}$ )

$$
I_{12}^{\prime}=I_{j_{1}, j_{2} J}^{\prime \prime}+\frac{2 j_{1}+1-2 p_{0}}{2 j_{1}-1} \frac{2 j_{2}+1-2 n_{0}}{2 j_{2}-1} I_{j_{1}, j_{2} J}^{\prime \prime \prime}
$$

where $I_{j_{1} j_{2} J}^{\prime \prime}$ and $I_{j_{1} j_{2} J}^{\prime \prime \prime}$ denote respectively the expectation values of the odd and even higher multipole components of the antisymmetrized Coulomb interaction in the state $\left|j_{1} j_{2} J\right\rangle$.

The odd and even higher multipole energies in the $\left|j_{1} j_{2} J\right\rangle$ state are given by

$$
\begin{aligned}
I_{j_{1} j_{2} J}^{\prime \prime}=-\frac{1}{2} \sum_{k} G^{k} & \left(n_{1} l_{1} j_{1}, n_{2} l_{2} j_{2}\right)\left\langle j_{1}\left\|C^{(k)}\right\| j_{2}\right\rangle^{2}\left[\left\{\begin{array}{lll}
j_{1} & j_{2} & J \\
j_{1} & j_{2} & k
\end{array}\right\}-\frac{\delta_{J, k}}{2 k+1}\right] \\
I_{j_{1} j_{2} J}^{\prime \prime \prime}= & \sum_{k \text { even } \neq 0} F^{k}\left(n_{1} l_{1} j_{1}, n_{2} l_{2} j_{2}\right)\left\langle j_{1}\left\|C^{(k)}\right\| j_{1}\right\rangle \\
& \times\left\langle j_{2}\left\|C^{(k)}\right\| j_{2}\right\rangle(-1)^{j_{1}+j_{2}+J}\left\{\begin{array}{lll}
j_{1} & j_{2} & J \\
j_{2} & j_{1} & k
\end{array}\right\} \\
& -\frac{1}{2} \sum_{k} G^{k}\left(n_{1} l_{1} j_{1}, n_{2} l_{2} j_{2}\right)\left\langle j_{1}\left\|C^{(k)}\right\| j_{2}\right\rangle^{2} \\
& \times\left[\left\{\begin{array}{lll}
j_{1} & j_{2} & J \\
j_{1} & j_{2} & k
\end{array}\right\}-\frac{2}{\left(2 j_{1}+1\right)\left(2 j_{2}+1\right)}+\frac{\delta_{J, k}}{2 k+1}\right] .
\end{aligned}
$$

From normalization considerations like those in sect. 4.3.1, the coefficients of all radial integrals are seen to be smaller than 1 . Since for two different shells $F^{k}>G^{k}$ and since intershell $F^{k}$ integrals are smaller than intrashell ones, one expects to have $\left|I_{j_{1} j_{2} J}^{\prime \prime}\right|<\left|I_{j_{1} j_{2} J}^{\prime \prime \prime}\right|<\pi$. Furthermore, the coefficient of the larger term $I_{j_{1} j_{2} J}^{\prime \prime \prime}$ in eq. (A.8) is a product of two fractions which is sometimes positive and sometimes negative and has a vanishing average value over all $j_{1}^{p_{0}} j_{2}^{n_{0}}$ contigurations. 
In conclusion, the average higher multipole interaction $I_{12}^{\prime}$ is expected to be smaller than the interaction parameters $\pi, d$ and $I_{12}^{0}$, eqs. (18)-(20).

These expectations are supported by the experimental data. The $I_{12}^{\prime}$ coefficient is hardly recognizable in the systematics in figs. 1 and 2, and attempts to retain it in the least squares adjustments resulted in statistically insignificant values in most non-diagonal shell regions. Consequently, we omitted it from the final eqs. (9), (11) and $(12 a, b)$.

This behavior of $I_{12}^{\prime}$ is in sharp contrast to the nuclear case, where a negative odd-odd interaction $I_{12}^{\prime}$ (the so-called ncutron-proton pairing) significantly affects the systematics of nuclear energies $\left.{ }^{26}\right)$. This means that for nuclear ground states one has $I_{12}^{\prime \prime}<I_{12}^{\prime \prime \prime}<0$ [see also ref. $\left.^{8}\right)$ ]. The difference between the nuclear and Coulomb case is presumably due to the similar magnitudes of the nuclear $F^{k}$ and $G^{k}$ integrals and their increase with $k$, and also to the existence of odd-tensor, non-central and spin-dependent components in the nuclear interaction (see also sect. 4.3.1).

\section{A.3. EXPERIMENTAL COULOMB DISPLACEMENT ENERGIES}

Table A.2 of experimental Coulomb displacement energies is arranged as in refs. ${ }^{15,16}$ ). The symbols $n$ and $c$, respectively, denote values added to or changed from the values given in these references. The CDE for the isotopes of $\mathrm{Cd}, \mathrm{Nd}, \mathrm{Ta}$, $\mathrm{Pt}$, Th and $\mathrm{U}$ are deduced directly from measured $Q$-values. All other CDE are calculated from the measured $E^{\times}$(IAS) with the use of ref. ${ }^{28}$ ).

\section{A.4. QUADRATIC APPROXIMATION IN THE MEAN TO $Z^{4 / 3}$}

The polynomials

$$
\begin{aligned}
& y_{0}(p)=\frac{1}{\sqrt{\delta}}, \\
& y_{1}(p)=\sqrt{\frac{3}{\delta}}\left(1-\frac{2}{\delta} p\right), \\
& y_{2}(p)=\sqrt{\frac{5}{\delta}}\left(1-\frac{6}{\delta} p+\frac{6}{\delta^{2}} p^{2}\right),
\end{aligned}
$$

define an orthonormal system of polynomials in $p$ in a given shell region of width $\delta=Z_{2}-Z_{1}$.

The function $Z^{4 / 3}=\left(Z_{1}+p\right)^{4 / 3}$ can be approximated to in the mean by an expansion in these polynomials

$$
\left(Z_{1}+p\right)^{4 / 3} \approx \sum_{i=0}^{2} c_{i} y_{i}(p),
$$

* The adoption of the r.h.s. of eq. (A.8) with two independent coefficients $I_{12}^{\prime \prime}$ and $I_{12}^{\prime \prime \prime}$ is impractical, as the $n$ and $p$ of the odd subshells are not well defined for mixed nuclear configurations. 
TABle A.2

Experimental Coulomb displacements energies

\begin{tabular}{|c|c|c|c|c|c|c|c|c|}
\hline & $A$ & $Z_{c}-Z_{>}$ & $T$ & $J^{\pi}$ & $\begin{array}{c}E_{\mathrm{x}}\left(Z_{<}\right) \\
(\mathrm{keV})\end{array}$ & $\begin{array}{c}E_{\mathrm{x}}\left(Z_{>}\right) \\
(\mathrm{keV})\end{array}$ & $\begin{array}{l}\text { CDE } \\
(\mathrm{keV})\end{array}$ & Refs. \\
\hline $\mathrm{n}$ & 22 & $\mathrm{~F}-\mathrm{Ne}$ & 2 & $4^{+}$ & g.s. & $14070 \pm 40$ & $3995 \pm 50$ & 27,28 ) \\
\hline$n$ & 22 & $\mathrm{Mg}-\mathrm{Al}$ & 2 & $4^{+}$ & $14044 \pm 15$ & g.s. & $5225 \pm 72$ & $29,28)$ \\
\hline $\mathrm{n}$ & 35 & $\mathrm{~K}-\mathrm{Ca}$ & $\frac{5}{2}$ & $\frac{1}{2}^{+}$ & $9053 \pm 45$ & g.s. & $7346 \pm 70$ & ${ }^{30,28}$ ) \\
\hline $\mathrm{n}$ & 41 & Ar-K & $\frac{5}{2}$ & $\frac{7}{2}^{-}$ & g.s. & $8349 \pm 15$ & $6639 \pm 15$ & 31.28 ) \\
\hline $\mathrm{n}$ & 48 & $\mathrm{Cr}-\mathrm{Mn}$ & 1 & $4^{+}$ & $5792 \pm 1$ & g.s. & $8597 \pm 22$ & $32,28)$ \\
\hline $\mathrm{n}$ & 57 & $\mathrm{Ni}-\mathrm{Cu}$ & $\frac{1}{2}$ & $\frac{3}{2}^{-}$ & g.s. & g.s. & $9509 \pm 50$ & $\left.{ }^{28}\right)$ \\
\hline c & 63 & $\mathrm{Cu}-\mathrm{Zn}$ & $\frac{5}{2}$ & $\frac{3}{2}^{-}$ & g.s. & $5495 \pm 7$ & $9644 \pm 8$ & 27,28 ) \\
\hline $\mathrm{n}$ & 95 & $\mathrm{Y}-\mathrm{Zr}$ & $\frac{17}{2}$ & $\frac{1}{2}^{-}$ & g.s. & $14980 \pm 20$ & $11317 \pm 21$ & 33,28 ) \\
\hline $\mathrm{c}$ & 110 & Cd-In & 7 & $0^{+}$ & g.s. & $8767 \pm 206$ & $13490 \pm 50$ & ${ }^{34}$ ) \\
\hline $\mathrm{n}$ & 116 & $\mathrm{Cd}-\mathrm{In}$ & 10 & $0^{+}$ & g.s. & $11990 \pm 50$ & $13240 \pm 50$ & ${ }^{34}$ ) \\
\hline $\mathrm{n}$ & 111 & In-Sn & $\frac{13}{2}$ & $\frac{9}{2}^{+}$ & g.s. & $10507 \pm 50$ & $13737 \pm 51$ & $35,28)$ \\
\hline c & 115 & In-Sn & $\frac{17}{2}$ & $\frac{9}{2}^{+}$ & g.s. & $13317 \pm 50$ & $13604 \pm 50$ & $\left.{ }^{35,28}\right)$ \\
\hline c & 117 & In-Sn & $\frac{19}{2}$ & $\frac{9}{2}^{+}$ & g.s. & $14151 \pm 50$ & $13479 \pm 50$ & $35,28)$ \\
\hline c & 119 & $\mathrm{In}-\mathrm{Sn}$ & $\frac{21}{2}$ & $\frac{9}{2}^{+}$ & g.s. & $14995 \pm 50$ & $13442 \pm 51$ & 35,28 ) \\
\hline $\mathrm{c}$ & 121 & In-Sn & $\frac{23}{2}$ & $\frac{9+}{2}$ & g.s. & $15953 \pm 50$ & $13373 \pm 57$ & $35,28)$ \\
\hline c & 123 & $\mathrm{In}-\mathrm{Sn}$ & $\frac{25}{2}$ & $\frac{9}{2}^{+}$ & g.s. & $16943 \pm 50$ & $13325 \pm 58$ & $35,28)$ \\
\hline $\mathrm{n}$ & 142 & $\mathrm{Nd}-\mathrm{Pm}$ & 11 & $0^{+}$ & g.s. & $9972 \pm 43$ & $15624 \pm 14$ & ${ }^{36}$ ) \\
\hline c & 144 & $\mathrm{Nd}-\mathrm{Pm}$ & 12 & $0^{+}$ & g.s. & $12372 \pm 12$ & $15487 \pm 11$ & ${ }^{36}$ ) \\
\hline $\mathrm{n}$ & 146 & $\mathrm{Nd}-\mathrm{Pm}$ & 13 & $0^{+}$ & g.s. & $13128 \pm 16$ & $15387 \pm 14$ & ${ }^{36}$ ) \\
\hline $\mathrm{n}$ & 148 & $\mathrm{Nd}-\mathrm{Pm}$ & 14 & $0^{+}$ & g.s. & $13970 \pm 18$ & $15296 \pm 13$ & ${ }^{36}$ ) \\
\hline $\mathbf{n}$ & 150 & $\mathrm{Nd}-\mathrm{Pm}$ & 15 & $0^{+}$ & g.s. & $14359 \pm 24$ & $15228 \pm 12$ & $\left.{ }^{36}\right)$ \\
\hline $\mathrm{c}$ & 181 & $\mathrm{Ta} \mathrm{W}$ & $\frac{35}{2}$ & $\frac{7}{2}^{+}$ & g.s. & $16572 \pm 8$ & $17542 \pm 6$ & ${ }^{37}$ ) \\
\hline $\mathrm{n}$ & 192 & $\mathrm{Pt}-\mathrm{Au}$ & 18 & $0^{+}$ & g.s. & $14124 \pm 17$ & $18422 \pm 5$ & $\left.{ }^{37}\right)$ \\
\hline $\mathrm{n}$ & 194 & $\mathrm{Pt}-\mathrm{Au}$ & 19 & $0^{+}$ & g.s. & $15086 \pm 13$ & $18360 \pm 3$ & ${ }^{37}$ ) \\
\hline $\mathrm{n}$ & 196 & $\mathrm{Pt}-\mathrm{Au}$ & 20 & $0^{+}$ & g.s. & $16023+7$ & $18310 \pm 3$ & ${ }^{37}$ ) \\
\hline $\mathrm{n}$ & 198 & $\mathrm{Pt}-\mathrm{Au}$ & 21 & $0^{+}$ & g.s. & $17134 \pm 7$ & $18242 \pm 3$ & $\left.{ }^{37}\right)$ \\
\hline $\mathrm{n}$ & 207 & $\mathrm{Tl}-\mathrm{Pb}$ & $\frac{45}{2}$ & $\frac{1}{2}^{+}$ & g.s. & $19280 \pm 60$ & $18635 \pm 60$ & $38,28)$ \\
\hline II & 230 & $\mathrm{Th}_{1}-\mathbf{P a}$ & 25 & $0^{+}$ & g.s. & $17765 \pm 17$ & $19857 \pm 16$ & $\left.{ }^{39}\right)$ \\
\hline $\mathrm{n}$ & 232 & Th-Pa & 26 & $0^{+}$ & g.s. & $18530 \pm 11$ & $19792 \pm 5$ & $\left.{ }^{39}\right)$ \\
\hline $\mathrm{n}$ & 234 & $\mathrm{U}-\mathrm{Np}$ & 25 & $0^{+}$ & g.s. & $17610 \pm 12$ & $20203 \pm 7$ & $\left.{ }^{39}\right)$ \\
\hline $\mathrm{n}$ & 236 & U-Np & 26 & $0^{+}$ & g.s. & $18434 \pm 56$ & $20145 \pm 26$ & $\left.{ }^{39}\right)$ \\
\hline c & 238 & $\mathrm{U}-\mathrm{Np}$ & 27 & $0^{+}$ & g.s. & $19117 \pm 8$ & $20045 \pm 7$ & 39) \\
\hline
\end{tabular}

where

$$
\begin{aligned}
& c_{0}=\frac{1}{\sqrt{\delta}} \frac{3}{7}\left(Z_{2}^{7 / 3}-Z_{1}^{7 / 3}\right), \\
& c_{1}=\sqrt{\frac{3}{\delta}}\left[\frac{9}{35 \delta}\left(Z_{2}^{10 / 3}-Z_{1}^{10 / 3}\right)-\frac{3}{7}\left(Z_{2}^{7 / 3}+Z_{1}^{7 / 3}\right)\right], \\
& c_{2}=\sqrt{\frac{5}{\delta}}\left[\frac{162}{455 \delta^{2}}\left(Z_{2}^{13 / 3}-Z_{1}^{13 / 3}\right)-\frac{27}{35 \delta}\left(Z_{2}^{10 / 3}+Z_{1}^{10 / 3}\right)+\frac{3}{7}\left(Z_{2}^{7 / 3}-Z_{1}^{7 / 3}\right)\right] .
\end{aligned}
$$


Rearranging eq. (A.14) one obtains eq. (37) with

$$
\begin{aligned}
& \alpha=\frac{1}{\sqrt{\delta}} c_{0}+\sqrt{\frac{3}{\delta}} c_{1}+\sqrt{\frac{5}{\delta}} c_{2}, \\
& \beta=-\frac{2}{\delta} \sqrt{\frac{3}{\delta}} c_{1}-\frac{6}{\delta} \sqrt{\frac{5}{\delta}} c_{2}, \\
& \gamma=\frac{6}{\delta^{2}} \sqrt{\frac{5}{\delta}} c_{2} .
\end{aligned}
$$

Using the quadratic approximation (37) one obtains for $\beta_{2}^{2}$ of eq. (50) the expression

$$
\beta_{2}^{2}\left(Z_{1}+p, A\right)=-4 \pi \frac{\left(c+\frac{1}{2} \pi\right)(1-\kappa \gamma)-d\left(Z_{1}+\frac{1}{2}-\frac{1}{2} \kappa(\beta+\gamma)\right)}{c+\frac{1}{2} \pi+\frac{1}{2} d(\delta-1)} \frac{p(\delta-p)}{\left(Z_{1}+p\right)^{2}}
$$

The r.h.s. of eq. (A.21) attains its maximum value in a given shell region at

$$
p_{\max }=\frac{\delta Z_{1}}{2 Z_{1}+\delta}
$$

The r.h.s. of eq. (A.22) agrees with the integral values of $p_{\max }$ from the second column of table 7 to better than \pm 0.5 in all shell regions, except for region $(6,6)$, where the two terms in the numerator of the r.h.s. of eq. (50) largely cancel for $p=p_{\max }$. The value of the r.h.s. of eq. (A.22) for regions $(6,6)$ and $(7,6)$ is 12.1 , as compared to the value 9 in table 7 .

On the other hand, the values of the r.h.s. of eq. (A.21) for $p=p_{\max }$ from eq. (A.22) agree with the values of $\beta_{2 \max }$ in the fourth column of table 7 from eq. (50) to better than 0.01 in all shell regions, except for region $(2,2)$ where the respective $\beta_{2 \max }$ values of eqs. (A.21) and (50) are 0.11 and 0.26 .

For convenience in calculations we give in table A.3 the numerical values of the coefficients $\alpha, \beta$ and $\gamma$ for the various shell regions.

TABLe A.3

Numerical values of the coefficients $\alpha, \beta$ and $\gamma$

\begin{tabular}{crcc}
\hline Major shell & \multicolumn{1}{c}{$\alpha$} & $\beta$ & $\gamma$ \\
\hline 2 & 2.471767 & 1.790024 & 0.078420 \\
3 & $\mathbf{1 5 . 9 3 8 2 1 5}$ & 2.733875 & 0.038844 \\
4 & 54.281730 & 3.629458 & 0.026770 \\
5 & 84.960218 & 4.085665 & 0.019448 \\
6 & 184.120597 & 4.943732 & 0.013671 \\
7 & 356.149987 & 5.820223 & 0.010085 \\
\hline
\end{tabular}




\section{References}

1) S. Liran and N. Zeldes, At. Data Nucl. Data Tables 17 (1976) 431

2) B.C. Carlson and I. Talmi, Phys. Rev. 96 (1954) 436

3) A. de-Shalit and I. Talmi, Nuclear shell theory (Academic Press, New York, 1963)

4) S. Liran, Ph.D. Thesis, Jerusalem, 1973 (in Hebrew), unpublished

5) E. Comay and J. Jänecke, Nucl. Phys. A410 (1983) 103

6) E.P. Wigner in Proc. Robert A. Welch Found. Conf. on chemical research I. The structure of the nucleus, ed. W.O. Milligan (The Robert A. Welch Foundation, Houston, 1958) 67

7) J. Jänecke, Isospin in nuclear physics, ed. D.H. Wilkinson (North-Holland, Amsterdam, 1969) 297

8) N. Zeldes, Nucl. Phys. 7 (1958) 27

9) K.T. Hecht, Nucl. Phys. A102 (1967) 11

10) I. Talmi, Nucl. Phys. A172 (1971) 1

11) K.T. Hecht, Nucl. Phys. A114 (1968) 280

12) S. Elitzur, M.Sc. Thesis, Jerusalem, 1971 (in Hebrew), unpublished

13) H.R. Collard, L.R.B. Elton and R. Hofstadter, Nuclear radii (Springer, Berlin, 1967)

14) H. de Vries, C.W. de Jager and C. de Vries, At. Data Nucl. Data Tables 36 (1987) 495

15) M.S. Antony, J. Britz, J.B. Bueb and A. Pape, At. Data Nucl. Data Tables 33 (1985) 447

16) M.S. Antony, J. Britz and A. Pape, At. Data Nucl. Data Tables 40 (1988) 9

17) J.D. Anderson, C. Wong and J.W. McClure, Phys. Rev. 138 (1965) B615

18) B.A. Brown, C.R. Bronk and P.F. Hodgson, J. of Phys. G10 (1984) 1683

19) E.R. Cohen and B.N. Taylor, Revs. Mod. Phys. 59 (1987) 1121

20) L.N. Cooper and E. Henley, Phys. Rev. 92 (1953) 801

21) L. Wilets, Theories of nuclear fission (Oxford, 1964)

22) S. Raman, C.H. Malarkey, W.T. Milner, C.W. Nestor Jr. and P.H. Stelson, At. Data Nucl. Data Tables 36 (1987) I

23) J. Jänecke, E.H.L. Aarts, A.G. Drentje, M.N. Harakeh and C. Gaarde, Nucl. Phys. A394 (1983) 39

24) J. Jänecke and E. Comay, Phys. Lett. B140 (1984) 1

25) J. Jänecke, Phys. Rev. 147 (1966) 735

26) N.B. Gove and M. Yamada, Nucl. Data A4 (1968) 237

27) W.J. Courtney and J.D. Fox, At. Data Nucl. Data Tables 15 (1975) 141

28) A.H. Wapstra, G. Audi and R. Hoekstra, At. Data Nucl. Data Tables 39 (1988) 281

29) M.D. Cable, J. Honkanen, R.F. Parry, H.M. Thierens, J.M. Wouters, Z.Y. Zhou and J. Cerny, Phys. Rev. C.26 (198?) 1778

30) J. Äystö, D. M. Moltz, X.J. Xu, J.E. Reiff and J. Cerny, Phys. Rev. Lett. 55 (1985) 1384

31) L.R. Medsker, H.T. Fortune and S.C. Headley, Phys. Rev. C12 (1975) 401

32) T. Sekine, J. Cerny, R. Kirchner, O. Klepper, V.T. Koslowsky, A. Plochocki, E. Roeckl, D. Schardt, B. Sherrill and B.A. Brown, Nucl. Phys. A467 (1987) 93

33) S. Galès, E. Hourani, S. Fortier, H. Laurent, J.M. Maison and J.P. Schapira, Nucl. Phys. A288 (1977) 201

34) T. Murakami, S. Nishihara, T. Nakagawa, S. Morita, H. Orihara, K. Maeda and K. Miura, Nucl. Phys. A377 (1982) 163

35) H. Taketani, M. Adachi, T. Matsuzaki, M. Matoba, N. Koori, T. Yamazaki, S. Morinobu, I. Katayama, M. Fujiwara, Y. Fujita and H. Ikegami, Phys. Lett. B90 (1980) z14

36) H.J. Hofmann, S. Brandenburg, P. Grasdijk, M.N. Harakeh, W.A. Sterrenburg and S.Y. van der Werf, Nucl. Phys. A433 (1985) 181 and private communication

37) G.W.R. Leibbrandt, Report KVI-115i (1986) p. 37 and private communication

38) G. Duhamel, G. Perrin, J. Chauvin, M. Buenerd, E. Gerlic, J. Guillot, J.P. Didelez, H. Langevin-Joliot and J. van de Wiele, Phys. Lett. B78 (1978) 213

39) G. Brower, Report KVI-000 (1989), p. 31 and private communication 